TRANSACTIONS OF THE

AMERICAN MATHEMATICAL SOCIETY

Volume 361, Number 3, March 2009, Pages 1543-1579

S 0002-9947(08)04630-8

Article electronically published on October 21, 2008

\title{
APPROXIMATION PROPERTIES ON INVARIANT MEASURE AND OSELEDEC SPLITTING IN NON-UNIFORMLY HYPERBOLIC SYSTEMS
}

\author{
CHAO LIANG, GENG LIU, AND WENXIANG SUN
}

\begin{abstract}
We prove that each invariant measure in a non-uniformly hyperbolic system can be approximated by atomic measures on hyperbolic periodic orbits. This contributes to our main result that the mean angle (Definition 1.10), independence number (Definition 1.6) and Oseledec splitting for an ergodic hyperbolic measure with simple spectrum can be approximated by those for atomic measures on hyperbolic periodic orbits, respectively. Combining this result with the approximation property of Lyapunov exponents by Wang and Sun, 2005 (Theorem 1.9), we strengthen Katok's closing lemma (1980) by presenting more extensive information not only about the state system but also its linearization.

In the present paper, we also study an ergodic theorem and a variational principle for mean angle, independence number and Liao's style number (Definition 1.3) which are bases for discussing the approximation properties in the main result.
\end{abstract}

\section{INTRODUCTION}

Let $f: M \rightarrow M$ be a $C^{1}$ diffeomorphism of a compact smooth Riemannian manifold $M$ and let $D f: T M \rightarrow T M$ be the tangent map on the tangent bundle $T M$. For any integer $\ell, 1 \leq \ell \leq \operatorname{dim} M=d$, we construct a bundle $\mathcal{U}_{\ell}^{\#}(M)=$ $\bigcup_{x \in M} \mathcal{U}_{\ell}^{\#}(x)$ of $\ell$-frames, where the fiber over $x$ is

$\mathcal{U}_{\ell}^{\#}(x)=\left\{\alpha=\left(u_{1}, u_{2}, \ldots, u_{\ell}\right) \in T_{x} M \times T_{x} M \times \cdots \times T_{x} M \mid\left\|u_{i}\right\|=1, i=1, \ldots, \ell\right.$, and $u_{1}, u_{2}, \ldots, u_{\ell}$ are linearly independent vectors $\}$.

Set $\mathcal{V}_{\ell}^{\#}(M)=\bigcup_{x \in M} \mathcal{V}_{\ell}^{\#}(x)$, where the fiber over $x$ is $\mathcal{V}_{\ell}^{\#}(x)=\left\{\alpha=\left(u_{1}, u_{2}, \ldots, u_{\ell}\right) \in T_{x} M \times T_{x} M \times \cdots \times T_{x} M \mid\left\|u_{i}\right\|=1, i=1, \ldots, \ell\right.$, and $u_{1}, u_{2}, \ldots, u_{\ell}$ are linearly dependent vectors $\}$.

Let $L_{\ell}^{\#}(M):=\mathcal{U}_{\ell}^{\#}(M) \cup \mathcal{V}_{\ell}^{\#}(M)$; then $L_{\ell}^{\#}(M)$ forms a compact metric space. Let $\pi: L_{\ell}^{\#}(M) \rightarrow M$ be the natural bundle projection. The tangent map $D f$ induces

Received by the editors March 2, 2007.

2000 Mathematics Subject Classification. Primary 37C40, 37D25, 37H15, 37A35.

Key words and phrases. Independence number, invariant measure, mean angle.

The first and second authors were supported by NNSFC(\# 10671006).

The third author was supported by NNSFC (\# 10231020, \# 10671006), the National Basic Research Program of China (973 Program)(\# 2006CB805900) and the Doctoral Education Foundation of China.

(C)2008 American Mathematical Society Reverts to public domain 28 years from publication 
a map $D^{\#} f$ on $L_{\ell}^{\#}(M)$ :

$$
\begin{gathered}
D^{\#} f: L_{\ell}^{\#}(M) \rightarrow L_{\ell}^{\#}(M), \\
D^{\#} f\left(u_{1}, u_{2}, \ldots, u_{\ell}\right)=\left(\frac{D f\left(u_{1}\right)}{\left\|D f\left(u_{1}\right)\right\|}, \frac{D f\left(u_{2}\right)}{\left\|D f\left(u_{2}\right)\right\|}, \ldots, \frac{D f\left(u_{\ell}\right)}{\left\|D f\left(u_{\ell}\right)\right\|}\right) .
\end{gathered}
$$

It is clear that

$$
\pi \circ D^{\#} f=f \circ \pi .
$$

For an $\ell$-frame $\alpha=\left(u_{1}, u_{2}, \ldots, u_{\ell}\right) \in \mathcal{U}_{\ell}^{\#}(x)$ at a point $x \in M$, we define $\operatorname{Vol}(\alpha)=\operatorname{Vol}\left(u_{1}, u_{2}, \ldots, u_{\ell}\right)$ as the volume of the parallelepiped generated by the vectors $u_{1}, u_{2}, \ldots, u_{\ell}$. More precisely, we choose an orthonormal $\ell$-frame $\beta=$ $\left(w_{1}, w_{2}, \ldots, w_{\ell}\right), w_{i} \in T_{x} M, \quad i=1, \ldots, \ell$, which generates the same linear subspace of $T_{x} M$ as $\alpha$ does, and we take a unique $\ell \times \ell$ matrix $A$ with $\alpha=\beta A$. Then we define the volume of $\alpha$ by

$$
\operatorname{Vol}(\alpha):=|\operatorname{det} A| .
$$

We remark that the volume $\operatorname{Vol}(\alpha)$ does not depend on the choice of $\beta$, since the determinate of a transition matrix between two orthonormal frames is \pm 1 . Hence, $\operatorname{Vol}(\alpha)$ is well defined.

Definition $1.1([6])$. A frame $\alpha=\left(u_{1}, u_{2}, \ldots, u_{\ell}\right) \in \mathcal{U}_{\ell}^{\#}(x), x \in M$, is called positive mean linearly independent if there is $\epsilon>0$ such that the characteristic function $\chi_{\alpha \epsilon}$ on the set

$$
\left\{n \in \mathbb{Z}^{+} \mid \operatorname{Vol}\left(D^{\#} f^{n}(\alpha)\right) \geq \epsilon\right\}
$$

has positive time mean; that is,

$$
\varlimsup_{n \rightarrow+\infty} \frac{1}{n} \sum_{i=0}^{n-1} \chi_{\alpha \epsilon}(i)>0 .
$$

If for any positive number $\epsilon$, the above supper limit is zero, $\alpha$ is called positive mean linearly dependent. Sometimes we write $\chi_{\alpha \epsilon}(i)$ as $\chi_{\alpha \epsilon}\left(D^{\#} f^{i}\right)$ to emphasize the $i-$ th iteration of $D^{\#} f$. Similarly, one can define negative mean linear independence and dependence by using the negative time mean

$$
\varlimsup_{n \rightarrow-\infty} \frac{1}{|n|} \sum_{i=0}^{n-1} \chi_{\alpha \epsilon}\left(D^{\#} f^{i}\right) .
$$

Let $\alpha=\left(u_{1}, \ldots, u_{\ell}\right) \in \mathcal{U}_{\ell}^{\#}$. Denote by $A(\alpha)$ the matrix $\left(\left\langle u_{i}, u_{j}\right\rangle\right)_{\ell \times \ell}$, where $\langle\cdot, \cdot\rangle$ is the inner product induced by the Riemannian metric. Let $\sigma(\alpha)$ denote the set of all eigenvalues of $A(\alpha)$ and let $\tau(\alpha)$ be the smallest eigenvalue. Note that $A(\alpha)$ is a real positive-definite symmetric matrix, therefore, $\sigma(\alpha) \subset(0,+\infty)$. Now we give an alternative description of mean linear independence considering the average change of $\tau(\alpha)$ over time instead of that of $\operatorname{Vol}(\alpha)$ (for the equivalence of these two definitions see Proposition 2.3).

Definition 1.2. A frame $\alpha \in \mathcal{U}_{\ell}^{\#}$ is positive mean linearly independent if and only if

$$
\varlimsup_{n \rightarrow+\infty} \frac{1}{n} \sum_{i=0}^{n-1} \tau\left(D^{\#} f^{i} \alpha\right)>0 .
$$

It is negative mean linearly independent if and only if

$$
\varlimsup_{n \rightarrow-\infty} \frac{1}{|n|} \Sigma_{i=0}^{n-1} \tau\left(D^{\#} f^{i} \alpha\right)>0 .
$$


Let $X$ be a metric space. We denote by $C^{b}(X, \mathbb{R})$ the set of all bounded continuous functions on $X$. Given a continuous map $g: X \rightarrow X$, we denote by $\mathcal{M}_{\text {erg }}(X, g)$ the set of all $g$-invariant and ergodic measures. Then we define for $m \in \mathcal{M}_{\text {erg }}(X, g)$,

$$
\begin{gathered}
Q_{m}(X, g):=\left\{x \in X \mid \lim _{n \rightarrow+\infty} \frac{1}{n} \sum_{i=0}^{n-1} \phi\left(g^{i}(x)\right)=\lim _{n \rightarrow-\infty} \frac{1}{n} \sum_{i=0}^{n-1} \phi\left(g^{i}(x)\right)\right. \\
\left.=\int_{X} \phi d m, \quad \forall \phi \in C^{b}(X, \mathbb{R})\right\} .
\end{gathered}
$$

We call $Q_{m}(X, g)$ the basin of $m$. By the Birkhoff Ergodic Theorem $Q_{m}(X, g)$ is an $m$-full measure set and $g\left(Q_{m}(X, g)\right)=Q_{m}(X, g)$. We can replace $C^{b}(X, \mathbb{R})$ by $C^{0}(X, \mathbb{R})$, the set of all continuous functions on $X$, in $Q_{m}$ when $X$ is compact.

Definition 1.3. (1) Let $x \in M$ and set

$$
k_{+}^{*}(x):=\max \left\{\ell \mid \exists \alpha \in \mathcal{U}_{\ell}^{\#}(x) \text {, s.t. } \alpha \text { is positive mean linearly independent }\right\}
$$

and

$$
k_{-}^{*}(x):=\max \left\{\ell \mid \exists \alpha \in \mathcal{U}_{\ell}^{\#}(x), \text { s.t. } \alpha \text { is negative mean linearly independent }\right\} .
$$

We call $k_{+}^{*}(x)$ and $k_{-}^{*}(x)$ the positive and the negative style number of $f$ at $x$, respectively.

(2) Let $F \subset M$ be an $f$-invariant subset. The positive style number $k_{+}^{*}(F)$ and the negative style number $k_{-}^{*}(F)$ of $F$ are defined as

$$
k_{+}^{*}(F):=\sup _{x \in F} k_{+}^{*}(x) \quad \text { and } \quad k_{-}^{*}(F):=\sup _{x \in F} k_{-}^{*}(x),
$$

respectively. The style number $k^{*}(F)$ of $F$ is defined by

$$
k^{*}(F):=\max \left\{k_{+}^{*}(F), k_{-}^{*}(F)\right\} .
$$

(3) Let $m \in \mathcal{M}_{\text {erg }}(M, f)$. The positive style number, the negative style number and the style number of $m$ are given by, respectively,

$$
k_{+}^{*}(m):=k_{+}^{*}\left(Q_{m}(M, f)\right), k_{-}^{*}(m):=k_{-}^{*}\left(Q_{m}(M, f)\right)
$$

and

$$
k^{*}(m):=\max \left\{k_{+}^{*}(m), k_{-}^{*}(m)\right\} .
$$

Definition $1.3(1)(2)$ are the diffeomorphism version of Liao's style number in 6 . Definition 1.3 (3), adapted by Dai 2], is a special case of Definition 1.3 (2) with $F=Q_{m}(M, f)$. One may replace $Q_{m}(M, f)$ by any invariant subset of $m$-total measure, for instance, $Q_{m}(M, f) \cap \operatorname{supp}(m)$, where $\operatorname{supp}(m)$ denotes the support of $m$.

The following is the diffeomorphism version of the main theorem concerning style number in [6].

Theorem 1.4 ([6]). Let $f: M \rightarrow M$ be a $C^{1}$ diffeomorphism of a compact smooth Riemannian manifold and let $F \subset M$ be a closed $f$-invariant set. Then

(1) $k_{-}^{*}(F)=k_{+}^{*}(F)=k^{*}(F) \triangleq k$.

(2) There is $x \in M$ and $\alpha \in \mathcal{U}_{k}^{\#}(x)$ such that the orbit $\operatorname{Orb}(x)$ is $f$-recurrent and

$$
\lim _{\epsilon \rightarrow 0} \varlimsup_{n \rightarrow+\infty} \frac{1}{n} \sum_{i=0}^{n-1} \chi_{\alpha \epsilon}\left(D^{\#} f^{i}\right)=\lim _{\epsilon \rightarrow 0} \varlimsup_{n \rightarrow-\infty} \frac{1}{|n|} \Sigma_{i=0}^{n-1} \chi_{\alpha \epsilon}\left(D^{\#} f^{i}\right)=1 .
$$


One result in the present paper is an ergodic theorem (Theorem 1.5) about mean linear independence and style number. Note that the basin of a given ergodic measure is contained in the Birkhoff center ([8], [19] and [20]) and thus always contains recurrent orbits. Hence Theorem 1.5 is a generalization of Theorem 1.4. Moreover, the upper limit in Theorem 1.4 could be exactly the limit.

Theorem 1.5. Let $f: M \rightarrow M$ be a $C^{1}$ diffeomorphism of a compact smooth Riemannian manifold. Let $m \in \mathcal{M}_{\text {erg }}(M, f)$. Then

$$
k_{-}^{*}(x)=k_{-}^{*}(m), \quad k_{+}^{*}(x)=k_{+}^{*}(m),
$$

for $m-$ a.e. $x \in M$ and

$$
k_{-}^{*}(m)=k_{+}^{*}(m)=k^{*}(m) \triangleq k .
$$

(2) For $m$ - a.e. $x \in M$ there is an orthonormal $k$-frame $\alpha \in \mathcal{U}_{k}^{\#}(x)$ such that

$$
\lim _{\epsilon \rightarrow 0} \lim _{n \rightarrow+\infty} \frac{1}{n} \sum_{i=0}^{n-1} \chi_{\alpha \epsilon}\left(D^{\#} f^{i}\right)=\lim _{\epsilon \rightarrow 0} \lim _{n \rightarrow-\infty} \frac{1}{|n|} \sum_{i=0}^{n-1} \chi_{\alpha \epsilon}\left(D^{\#} f^{i}\right)=1 .
$$

(3) There exists $\mu \in \mathcal{M}_{\text {erg }}\left(\mathcal{U}_{k}^{\#}, D^{\#} f\right), \pi_{*}(\mu)=m$, such that the set of all $\alpha \in \mathcal{U}_{k}^{\#}$ satisfying (1.1) is $\mu$-full measure.

Definition 1.6. (1) Let $\alpha \in \mathcal{U}_{\ell}^{\#}$. We call the supper limit

$$
\tilde{\tau}(\alpha):=\varlimsup_{n \rightarrow+\infty} \frac{1}{n} \sum_{i=0}^{n-1} \tau\left(D^{\#} f^{i} \alpha\right)
$$

the independence number for $\alpha$.

(2) If $\mathcal{M}_{\text {erg }}\left(\mathcal{U}_{\ell}^{\#}, D^{\#} f\right) \neq \emptyset$, we define for $\mu \in \mathcal{M}_{\text {erg }}\left(\mathcal{U}_{\ell}^{\#}, D^{\#} f\right)$

$$
\tilde{\tau}(\mu):=\sup \left\{\tilde{\tau}(\alpha) \mid \alpha \in Q_{\mu}\left(\mathcal{U}_{\ell}^{\#}, D^{\#} f\right)\right\}
$$

and call $\tilde{\tau}(\mu)$ the independence number for $\mu$.

For $\alpha \in \mathcal{V}_{\ell}^{\#}$, we define $\tilde{\tau}(\alpha)=0$. Then $\tilde{\tau}(\cdot)$ is a function from $L_{\ell}^{\#}$ to $\mathbb{R}$.

Remark. Replacing $\tau\left(D^{\#} f^{i} \alpha\right)$ by $\operatorname{Vol}\left(D^{\#} f^{i} \alpha\right)$ in Definition 1.6 one gets an alternative description of independence numbers (ref. Proposition 2.3).

The next theorem is a variational principle for style numbers and independence numbers, which asserts that the biggest style number can be achieved at an ergodic measure (on the state manifold) and at the same time, and that the largest independence number can be achieved at an ergodic measure (on the unit frame bundle) covering the one with the biggest style number.

Theorem 1.7. Let $f: M \rightarrow M$ be a $C^{1}$ diffeomorphism on a compact smooth Riemannian manifold and let $k=k^{*}(M)$. Then there exist $m_{0} \in \mathcal{M}_{\text {erg }}(M, f)$ and $\nu_{0} \in \mathcal{M}_{\text {erg }}\left(\mathcal{U}_{k}^{\#}, D^{\#} f\right)$, with $\pi_{*}\left(\nu_{0}\right)=m_{0}$, such that

$$
k^{*}(x)=k^{*}\left(m_{0}\right)=\max \left\{k^{*}(m) \mid m \in \mathcal{M}_{\text {erg }}(M, f)\right\}=k^{*}(M), \quad m_{0}-a . a . x \in M
$$

and

$\tilde{\tau}(\beta)=\tilde{\tau}\left(\nu_{0}\right)=\sup \left\{\tilde{\tau}(\mu) \mid \mu \in \mathcal{M}_{\text {erg }}\left(\mathcal{U}_{k}^{\#}, D^{\#} f\right)\right\}=\sup _{\alpha \in L_{k}^{\#}(M)} \tilde{\tau}(\alpha), \quad \nu_{0}-a . a . \beta \in \mathcal{U}_{k}^{\#}$. 
We will present some examples in Section 2.2 to compare the style number with the number of Lyapunov exponents and the dimension of the state manifold. In Sections 2.3 and 2.4 we will prove Theorem 1.5] and Theorem 1.7, respectively. In 2. Dai obtained certain results concerning the style number in the direction of Theorem 1.5 and Theorem 1.7 for $C^{1}$ vector fields.

Theorem [1.5 and Theorem 1.7 will contribute to the proof of our main result, Theorem 1.12. Another crucial result in its proof is the following theorem on measure approximation. We denote by $\mathcal{M}_{H P}(f)$ the set of atomic measures on hyperbolic periodic orbits.

Theorem 1.8. Let $f: M \rightarrow M$ be a $C^{1+\alpha}(\alpha>0)$ diffeomorphism on a compact manifold. Suppose that $f$ preserves an ergodic hyperbolic measure $\tilde{\omega}$. Then there exists an $f$-invariant set $\tilde{\Lambda}$ of $\tilde{\omega}$-full measure such that $\mathcal{M}_{H P}(f)$ is dense in $\mathcal{M}_{\text {inv }}(\tilde{\Lambda})$, the set of all $f$-invariant measures supported by $\tilde{\Lambda}$.

Theorem 1.8 is a generalization of Sigmund's result for uniform hyperbolicity 14 and Hirayama's result for mixing non-uniform hyperbolicity 3 .

We will prove Theorem 1.8 in Section 3.

Let us recall the Oseledec theorem and give some necessary notions.

The Oseledec Theorem ([9]). Let $f$ be a $C^{1}$ diffeomorphism of a compact $d$ dimensional Riemannian manifold $M$ preserving an ergodic measure $\tilde{\omega}$. Then there exist

(a) real numbers $\lambda_{1}<\cdots<\lambda_{s}(s \leq d)$;

(b) positive integers $n_{1}, \ldots, n_{s}$, satisfying $n_{1}+\cdots+n_{s}=d$;

(c) a Borel set $O(\tilde{\omega})$, called an Oseledec basin of $\tilde{\omega}$, satisfying $f(O(\tilde{\omega}))=O(\tilde{\omega})$ and $\tilde{\omega}(O(\tilde{\omega}))=1$;

(d) a measurable splitting, called an Oseledec splitting, $T_{x} M=E_{1}(x) \oplus \cdots \oplus$ $E_{s}(x)$ with $\operatorname{dim}_{i}(x)=n_{i}$ and $D f\left(E_{i}(x)\right)=E_{i}(f x)$,

such that

$$
\lim _{n \rightarrow \pm \infty} \frac{\log \left\|D f^{n} v\right\|}{n}=\lambda_{i}
$$

for all $x \in O(\tilde{\omega}), v \in E_{i}(x), i=1,2, \ldots, s$.

(a) and (d) in the Oseledec Theorem allow us to arrange the Oseledec splitting according to the increasing order of the Lyapunov exponents. To avoid excessive terminology, we will arrange the Oseledec splitting at every point in the Oseledec basin in this way throughout this paper without explanation. We call the measure $\tilde{\omega}$ hyperbolic if none of its Lyapunov exponents is zero. The following theorem is the main theorem in [18.

Theorem 1.9 ([18]). Let $M$ be a compact d-dimensional Riemannian manifold. Let $f: M \rightarrow M$ be a $C^{1+\alpha}$ diffeomorphism, and let $\tilde{\omega}$ be an ergodic hyperbolic measure with Lyapunov exponents $\lambda_{1} \leq \cdots \leq \lambda_{r}<0<\lambda_{r+1} \leq \cdots \leq \lambda_{d}$. Then the Lyapunov exponents of $\tilde{\omega}$ can be approximated by Lyapunov exponents of hyperbolic periodic orbits. More precisely, for any $\epsilon>0$, there exists a hyperbolic periodic point $z$ with Lyapunov exponents $\lambda_{1}^{z} \leq \cdots \leq \lambda_{d}^{z}$ such that $\left|\lambda_{i}-\lambda_{i}^{z}\right|<\epsilon, i=1, \ldots, d$.

Definition 1.10. Let $E$ and $F$ be two $D f$-invariant sub-bundles of $T M$. The angle between $E(x)$ and $F(x), x \in M$, is defined as follows:

$$
\sin \angle(E(x), F(x)):=\inf _{0 \neq u \in E(x), 0 \neq v \in F(x)} \sin \angle(u, v)=\inf _{0 \neq u \in E(x), 0 \neq v \in F(x)} \frac{\|u \wedge v\|}{\|u\|\|v\|},
$$


where $\wedge$ denotes the wedge product. We call

$$
m \angle(E(x), F(x)):=\lim _{n \rightarrow+\infty} \frac{1}{n} \sum_{i=0}^{n-1} \angle\left(D f^{i} E(x), D f^{i} F(x)\right)
$$

the mean angle between $E$ and $F$ at $x$.

Suppose that $f$ preserves an ergodic measure $\tilde{\omega}$ with an Oseledec splitting

$$
T_{x} M=E_{1}(x) \oplus \cdots \oplus E_{s}(x), \quad s \leq d=\operatorname{dim} M,
$$

$x \in O(\tilde{\omega})$. By the Birkhoff Ergodic Theorem there is an $\tilde{\omega}$-full measure subset in $O(\tilde{\omega})$ such that every point $x$ in this subset satisfies

$$
m \angle\left(E_{i}(x), E_{j}(x)\right)=\int \angle\left(E_{i}(y), E_{j}(y)\right) d \tilde{\omega}(y) .
$$

We call $\int \angle\left(E_{i}(y), E_{j}(y)\right) d \tilde{\omega}(y)$ the mean angle between $E_{i}:=\bigcup_{x \in O(\tilde{\omega})} E_{i}(x)$ and $E_{j}:=\bigcup_{x \in O(\tilde{\omega})} E_{j}(x)$ and write it as $m \angle_{\tilde{\omega}}\left(E_{i}, E_{j}\right), \forall 1 \leq i \neq j \leq d$.

If, in addition, $\tilde{\omega}$ has $d$ different Lyapunov exponents, namely $\tilde{\omega}$ has a simple spectrum, the Oseledec splitting will be

$$
T_{x} M=E_{1}(x) \oplus \cdots \oplus E_{d}(x), \quad x \in O(\tilde{\omega}) .
$$

For $x \in O(\tilde{\omega})$, we define the independence number of $x$ by the independence number of frames at $x$ whose elements are all on different invariant bundles. More precisely, take $\alpha=\left(u_{1}, u_{2}, \ldots, u_{d}\right) \in \mathcal{U}_{d}^{\#}$, where $u_{i} \in E_{i}(x), i=1,2, \ldots, d$. Then we define

$$
\tau(x):=\tau(\alpha),
$$

the smallest eigenvalue of $A(\alpha)$. Clearly, $\tau(x)$ is well defined for $x \in O(\tilde{\omega})$. Moreover, by the Birkhoff Ergodic Theorem, the equation

$$
\lim _{n \rightarrow+\infty} \frac{1}{n} \sum_{i=0}^{n-1} \tau\left(f^{i} x\right)=\int \tau(y) d \tilde{\omega}(y)
$$

holds on an $\tilde{\omega}$-full measure subset of $O(\tilde{\omega})$. Therefore, we can define the independence number of $\tilde{\omega}$ by

$$
\tilde{\tau}(\tilde{\omega}):=\int \tau(y) d \tilde{\omega}(y) .
$$

Assume there is another ergodic hyperbolic measure $\tilde{\omega}^{\prime}$ with simple spectrum. We split the tangent bundle $T M$ into invariant bundles

$$
T_{y} M=E_{1}(y) \oplus E_{2}(y) \oplus \cdots \oplus E_{d}(y), \quad y \in O\left(\tilde{\omega}^{\prime}\right) .
$$

Additionally, we assume that $\tilde{\omega}$ and $\tilde{\omega}^{\prime}$ have the same number of negative Lyapunov exponents (and thus the same number of positive ones). Under these assumptions we further describe the approximation of Oseledec splittings. Remember both (1.2) and (1.3) are arranged according to the increasing order of Lyapunov exponents.

Definition 1.11. Let $\eta>0$. The Oseledec splitting (1.2) of $\tilde{\omega}$ is $\eta$ approximated by (1.3) of $\tilde{\omega}^{\prime}$ if there exists a measurable subset $A$ satisfying:

(1) $\tilde{\omega}(A)>1-\eta$ 
(2) for any $x \in A$ and any frame $\alpha=\left(u_{1}, \ldots, u_{d}\right) \in \mathcal{U}_{d}^{\#}(x)$ with $u_{i} \in$ $E_{\sigma(i)}(O(\tilde{\omega}))$, there exist a point $z=z(x) \in \operatorname{Supp}\left(\tilde{\omega}^{\prime}\right) \cap O\left(\tilde{\omega}^{\prime}\right)$ and a frame $\beta=\left(v_{1}, \ldots, v_{d}\right) \in \mathcal{U}_{d}^{\#}(z)$ with $v_{i} \in E_{\sigma(i)}\left(O\left(\tilde{\omega}^{\prime}\right)\right)$, such that

$$
\operatorname{dist}(\alpha, \beta)<\eta,
$$

where $\sigma:\{1, \ldots, d\} \rightarrow\{1, \ldots, d\}$ is a permutation, and dist denotes the metric on the Grassman bundle.

This definition does not depend on the choice of $\alpha$ or $\beta$. Now we state our main result of this paper.

Theorem 1.12. Let $f: M \rightarrow M$ be a $C^{1+\alpha}$ diffeomorphism preserving an ergodic hyperbolic measure $\tilde{\omega}$ which has a simple spectrum and denote $d=\operatorname{dim} M$. Let

$$
T_{\Lambda} M=E_{1}(\Lambda) \oplus E_{2}(\Lambda) \oplus \cdots \oplus E_{d}(\Lambda)
$$

be the Oseledec splitting of $\tilde{\omega}$ arranged according to the increasing order of Lyapunov exponents of $\tilde{\omega}$, where $\Lambda=\bigcup_{k \geq 1} \Lambda_{k}$ is the Pesin set associated with $\tilde{\omega}$ (see Section 3 for a definition). Given $\varepsilon>0$, there is a hyperbolic periodic orbit Orb $(z, f)$ together with its Oseledec splitting

$$
T_{z} M=E_{1}(z) \oplus E_{2}(z) \oplus \cdots \oplus E_{d}(z)
$$

at $z$ arranged according to the increasing order of Lyapunov exponents of the orbit $\operatorname{Orb}(z)$ such that the atomic measure $\omega_{z}$ supported on $\operatorname{Orb}(z, f)$ satisfies the following properties:

(1) Mean angles of $\tilde{\omega}$ and of $\omega_{z}$ are $\varepsilon-$ close; that is,

$$
\left|m \angle_{\tilde{\omega}}\left(E_{i}(\Lambda), E_{j}(\Lambda)\right)-m \angle_{\omega_{z}}\left(E_{i}(\operatorname{Orb}(z)), E_{j}(\operatorname{Orb}(z))\right)\right|<\varepsilon, \quad \forall 1 \leq i \neq j \leq d .
$$

(2) Independence numbers of $\tilde{\omega}$ and of $\omega_{z}$ are $\varepsilon$-close; that is,

$$
\left|\tilde{\tau}(\tilde{\omega})-\tilde{\tau}\left(\omega_{z}\right)\right|<\varepsilon \text {. }
$$

(3) The Oseledec splitting of $\tilde{\omega}$ is $\varepsilon$-approximated by that of $\omega_{z}$.

Theorem 1.9 implies that if $\tilde{\omega}$ has a simple spectrum, so do the atomic measures on the hyperbolic periodic orbits chosen in the theorem. It motivates us to investigate more approximation properties of mean angle, independence number and Oseledec splitting than we listed in Theorem 1.12 Observe that the Oseledec splitting is continuous neither with state points $x \in M$ nor with measures, and thus Theorem 1.9 cannot imply the approximation properties mentioned. A new approach would be required to prove Theorem 1.12 .

Here is how the proof goes. Let $f: M \rightarrow M$ be a $C^{1+\alpha}$ diffeomorphism on a compact manifold of dimension $d$, preserving an ergodic hyperbolic measure $\tilde{\omega}$ with a simple spectrum. We first choose a sequence of hyperbolic periodic orbits so that the $i$-th biggest exponent of every periodic orbit is close to the $i$-th biggest one of $\tilde{\omega}, 1 \leq i \leq d$ (see Theorem [1.9), and moreover, the atomic measures supported on these periodic orbits converge to $\tilde{\omega}$ (see Theorem 1.8). This is a crucial step in the proof since therefore we can transfer the properties of $\tilde{\omega}$ onto periodic orbits which are more flexible than $\tilde{\omega}$ itself. For each atomic measure, we can construct an ergodic measure on $\mathcal{U}_{k}^{\#}$ to cover it, where $k$ is the style number. This is from Theorem 2.7. Then we get a collection of ergodic measures on the bundle. Each of the limit measures of this collection covers $\tilde{\omega}$. Note that $\mathcal{U}_{k}^{\#}$ is not compact, and hence the case that no limit measure is supported on $\mathcal{U}_{k}^{\#}$ may happen. The 
key observation that helps us to overcome this obstacle is that a limit measure can be decomposed into the sum of finitely many ergodic measures on $\mathcal{U}_{k}^{\#}$ covering $\tilde{\omega}$ due to Sun and Vargas in [17]. Moreover, we show that not more than $2^{d}$ of these have effects and are of the same type; that is, in every direction the integral Lyapunov exponents with respect to all these measures are equal (see Definition 4.2). That two ergodic measures are of the same type implies that they play the same role in discussing a mean angle of $\tilde{\omega}$ between two given directions. Thus we focus on these not more than $2^{d}$ measures on $\mathcal{U}_{k}^{\#}$ covering $\tilde{\omega}$ and the mean angles of their generic frames. In this way we complete the verification of the approximation property about a mean angle. This analysis also helps us prove other approximation properties.

Finally we summarize these results to obtain an extensive version of Katok's closing lemma.

Theorem 1.13. Let $f: M \rightarrow M$ be a $C^{1+\alpha}$ diffeomorphism preserving an ergodic hyperbolic measure $\tilde{\omega}$ and denote $d=\operatorname{dim} M$. Let

$$
T_{\Lambda} M=E_{1}(\Lambda) \oplus E_{2}(\Lambda) \oplus \cdots \oplus E_{s}(\Lambda), \quad s \leq d,
$$

be the Oseledec splitting arranged according to the increasing order of Lyapunov exponents of $\tilde{\omega}$, where $\Lambda=\bigcup_{k \geq 1} \Lambda_{k}$ is the Pesin set associated with $\tilde{\omega}$. Given $\varepsilon>0$

(a) for any integer $k>0$, there is a number $\beta=\beta(k, \varepsilon)>0$ with the property that if $x, f^{p}(x) \in \Lambda_{k}$, and $d\left(x, f^{p} x\right)<\beta$ for some positive integer $p$, then there exists a unique hyperbolic periodic point $z \in M$ with period $p$, such that $d(z, x)<\varepsilon ;$ and

(b) there is an Oseledec splitting $T_{z} M=E_{1}(z) \oplus E_{2}(z) \oplus \cdots \oplus E_{s}(z)$ at $z$, such that the Lyapunov exponents of $z$ on $E_{i}(z)$ are $\varepsilon-$ close to the Lyapunov exponents of $\tilde{\omega}$ on $E_{i}(\Lambda)$ and $\operatorname{dim} E_{i}(z)=\operatorname{dim} E_{i}(\Lambda), \forall i=1,2, \ldots, s$; and

(c) let $\omega_{z}:=\frac{1}{p} \sum_{i=1}^{p} \delta_{f^{i} z}$ denote the atomic measure on the periodic orbit orb $(z)$. The distance between $\tilde{\omega}$ and $\omega_{z}$ is less than $\varepsilon$ in weak* topology;

If in addition $\tilde{\omega}$ has a simple Lyapunov spectrum, the following holds:

(d) mean angles of $\tilde{\omega}$ and of $\omega_{z}$ are $\varepsilon-$ close; that is,

$\left|m \angle_{\tilde{\omega}}\left(E_{i}(\Lambda), E_{j}(\Lambda)\right)-m \angle_{\omega_{z}}\left(E_{i}(\operatorname{Orb}(z)), E_{j}(\operatorname{Orb}(z))\right)\right|<\varepsilon, \quad \forall 1 \leq i \neq j \leq d ;$

(e) independence numbers of $\tilde{\omega}$ and of $\omega_{z}$ are $\varepsilon-$ close; that is,

$$
\left|\tilde{\tau}(\tilde{\omega})-\tilde{\tau}\left(\omega_{z}\right)\right|<\varepsilon
$$

(f) the Oseledec splittings of $\tilde{\omega}$ are $\varepsilon$-approximated by that of $\omega_{z}$.

Theorem 1.13 includes broader information than the original closing lemma of Katok [4, since it discusses not only the approximation property on the manifold but also the interrelated properties on its tangent bundles.

\section{Style Number AND indePEndEnCE NUmber}

2.1. Mean linear independence. We will prove the equivalence of Definition 1.1 and Definition 1.2 in this subsection.

Lemma 2.1. Let $\alpha \in \mathcal{U}_{\ell}^{\#}$; then

$$
\frac{\operatorname{det} A(\alpha)}{\ell^{\ell-1}} \leq \tau(\alpha) \leq(\operatorname{det} A(\alpha))^{\frac{1}{\ell}} \leq \ell^{\frac{1}{\ell}} .
$$


Proof. Let $\sigma(\alpha)=\left\{\tau_{i}\right\}_{i=1}^{\ell}$ with $\tau_{1} \geq \tau_{2} \geq \cdots \geq \tau_{\ell}=\tau(\alpha)$. Since $\operatorname{det} A(\alpha)=$ $\Pi_{i=1}^{\ell} \tau_{i} \geq(\tau(\alpha))^{\ell}$, then $\tau(\alpha) \leq(\operatorname{det} A(\alpha))^{\frac{1}{\ell}}$. Write $A(\alpha)$ as $\left(a_{i j}\right)_{\ell \times \ell}$ and let $\|A\|=$ $\max _{1 \leq i \leq \ell} \Sigma_{j=1}^{\ell}\left|a_{i j}\right|$. Then $\|A(\alpha)\| \leq \ell$. For each eigenvalue $\tau_{i} \in \sigma(\alpha)$, take an eigenvector $u$ with $\|u\|=1$. Then

$$
\tau_{i}=\left\|\tau_{i} u\right\|=\|A(\alpha) u\| \leq\|A(\alpha)\|\|u\|=\|A(\alpha)\| \leq \ell .
$$

So we have

and thus

$$
\operatorname{det} A(\alpha)=\Pi_{i=1}^{\ell} \tau_{i}=\tau(\alpha) \Pi_{i=1}^{\ell-1} \tau_{i} \leq \ell^{\ell-1} \tau(\alpha)
$$

$$
\frac{\operatorname{det} A(\alpha)}{\ell^{\ell-1}} \leq \tau(\alpha) \leq(\operatorname{det} A(\alpha))^{\frac{1}{\ell}} \leq \ell^{\frac{1}{\ell}}
$$

The proof of the following lemma is straightforward.

Lemma 2.2. Let $x \in M$ and $\alpha=\left(u_{1}, \ldots, u_{\ell}\right), \beta=\left(v_{1}, \ldots, v_{\ell}\right) \in \mathcal{U}_{\ell}^{\#}(x)$, where $\beta$ is an orthonormal frame. Suppose that $\alpha$ and $\beta$ generate the same subspace of $T_{x} M$ and $\alpha=\beta A$; then $\operatorname{det} A(\alpha)=(\operatorname{det} A)^{2}=\operatorname{Vol}(\alpha)^{2}$.

Proposition 2.3. Definition 1.1 and Definition 1.2 are equivalent.

Proof. Suppose that a frame $\alpha \in \mathcal{U}_{\ell}^{\#}$ satisfies

$$
\tilde{\tau}(\alpha)=\varlimsup_{n \rightarrow+\infty} \frac{1}{n} \sum_{i=0}^{n-1} \tau\left(D^{\#} f^{i} \alpha\right)>0 .
$$

Then there exist a subsequence $\left\{n_{j}\right\}_{j=1}^{+\infty}$ and a real $1 \geq \delta>0$ such that

$$
\lim _{j \rightarrow+\infty} \frac{1}{n_{j}} \Sigma_{i=0}^{n_{j}-1} \tau\left(D^{\#} f^{i} \alpha\right)=2 \delta .
$$

For any $0 \leq \mu \leq 1$, set

$$
N(\mu):=\#\left\{0 \leq i \leq n_{j} \mid \tau\left(D^{\#} f^{i} \alpha\right) \geq \mu \delta\right\}
$$

Note that $\tau\left(D^{\#} f^{i} \alpha\right) \leq \ell^{\frac{1}{\ell}} \leq 2$ by Lemma 2.1] so we have

$$
\frac{\left(n_{j}-N(\mu)\right) \mu \delta+2 N(\mu)}{n_{j}}>\delta
$$

as $j$ is large enough. Let $\iota(\mu):=\frac{N(\mu)}{n_{j}}$; then $\iota(\mu)>\frac{\delta-\mu \delta}{2-\mu \delta}$. Specially, for $\mu=\frac{1}{2}$, it holds that $\iota\left(\frac{1}{2}\right)>\frac{\delta}{4-\delta}$. Take $\epsilon:=\left(\frac{1}{2} \delta\right)^{\frac{\ell}{2}}$; then by Lemma 2.1 and Lemma 2.2, we have

$$
\begin{aligned}
& \#\left\{0 \leq i \leq n_{j} \mid \operatorname{Vol}\left(D^{\#} f^{i} \alpha\right) \geq \epsilon\right\} \\
\geq & \#\left\{0 \leq i \leq n_{j} \mid \tau\left(D^{\#} f^{i} \alpha\right) \geq \frac{1}{2} \delta\right\} \\
= & N\left(\frac{1}{2}\right)
\end{aligned}
$$

Thus

and hence,

$$
\frac{1}{n_{j}} \Sigma_{i=0}^{n_{j}-1} \chi_{\alpha \epsilon}\left(D^{\#} f^{i}\right) \geq \frac{N\left(\frac{1}{2}\right)}{n_{j}}=\iota\left(\frac{1}{2}\right)>\frac{\delta}{4-\delta},
$$

$$
\varlimsup_{n \rightarrow+\infty} \frac{1}{n} \sum_{i=0}^{n-1} \chi_{\alpha \epsilon}\left(D^{\#} f^{i}\right)>0
$$

So Definition 1.2 implies Definition 1.1. 
On the contrary, for a frame $\alpha \in \mathcal{U}_{\ell}^{\#}$ with

$$
\varlimsup_{n \rightarrow+\infty} \frac{1}{n} \sum_{i=0}^{n-1} \chi_{\alpha \epsilon}\left(D^{\#} f^{i}\right)>0,
$$

there exist a subsequence $\left\{n_{j}\right\}_{j=1}^{+\infty}$ and a real $1 \geq \delta>0$ such that

$$
\lim _{j \rightarrow+\infty} \frac{1}{n_{j}} \Sigma_{i=0}^{n_{j}-1} \chi_{\alpha \epsilon}\left(D^{\#} f^{i}\right)=2 \delta .
$$

By Lemma 2.1 and Lemma 2.2 we have

$$
\#\left\{0 \leq i \leq n_{j} \mid \tau\left(D^{\#} f^{i} \alpha\right) \geq \frac{\epsilon^{\frac{1}{2}}}{\ell^{\ell-1}}\right\} \geq \#\left\{0 \leq i \leq n_{j} \mid \operatorname{Vol}\left(D^{\#} f^{i} \alpha\right) \geq \epsilon\right\} .
$$

So

$$
\frac{1}{n_{j}} \Sigma_{i=0}^{n_{j}-1} \tau\left(D^{\#} f^{i} \alpha\right) \geq \frac{\epsilon^{\frac{1}{2}}}{\ell^{\ell-1}} \delta>0
$$

as $j$ is large enough. Hence,

$$
\varlimsup_{n \rightarrow \infty} \frac{1}{n} \sum_{i=0}^{n-1} \tau\left(D^{\#} f^{i} \alpha\right)>0 .
$$

Thus Definition 1.1 implies Definition 1.2 ,

2.2. Style number, number of Lyapunov exponents and dimension of state manifold. We will compare the style number of a diffeomorphism with the number of its Lyapunov exponents and the dimension of the state manifold in this subsection.

There are many cases where the style number coincides with the number of Lyapunov exponents, for instance, the well-known Thom automorphism on $\mathbb{T}^{2}$. In the following we present our first example which shows that the two numbers are different.

Example 1. Consider a $C^{1}$ diffeomorphism $f$ on a 2-dimensional manifold $M$ and a periodic orbit $\operatorname{orb}(x, f)=\left\{x, f(x), \ldots, f^{n-1}(x)\right\}$ satisfying $D_{x} f^{n}=3 I_{2 \times 2}$ : $T_{x} M \rightarrow T_{x} M$, where $I_{2 \times 2}$ denotes the $2 \times 2$ unit matrix. Let $m$ be the atomic measure on this orbit. Then $\log 3$ is the unique Lyapunov exponent. Take and fix an orthonormal frame $\alpha=\left(e_{1}(x), e_{2}(x)\right)$ in $T_{x} M$. Since $\operatorname{Vol}\left(D^{\#} f^{n}\left(e_{1}(x), e_{2}(x)\right)\right)=1$ $\forall n \in \mathbb{Z}^{+}$, then $k^{*}(m)=2$.

The style number of a diffeomorphism is not less than the number of its Lyapunov exponents, since we can construct a mean linearly independent frame each of whose vectors is contained in some invariant bundle by the Oseledec Theorem. Our next example, an adaption of Liao's example in [6], shows that the style number may be strictly smaller than the dimension of $M$.

Example 2. Let $\phi: \mathbb{R} \rightarrow \mathbb{R}$ be a $C^{\infty}$ bump function which satisfies

$$
\phi(x)=0 \quad \text { for } \quad x \leq \frac{1}{2} \quad \text { or } \quad x \geq \frac{3}{2}
$$

and

$$
\left.\frac{d \phi}{d x}\right|_{x=1}=b \neq 0
$$

Define $\psi: \mathbb{R}^{2} \rightarrow \mathbb{R}^{2},(r, \theta) \rightarrow(r, \theta+\phi(r))$, in polar coordinates. Extend $\psi$ to the sphere $\mathbb{S}^{2}=\mathbb{R}^{2} \cup\{\infty\}$ and thus obtain a new diffeomorphism $f: \mathbb{S}^{2} \rightarrow \mathbb{S}^{2}$, with $f(z)=\psi(z), \quad z \in \mathbb{R}^{2}$. Then $\Gamma: r=1$ is an $f$-invariant closed set in the manifold 
$\mathbb{S}^{2}$, and for any point $z \in \Gamma$ and any orthonormal frame $\alpha_{z}=\left(u_{z}, u_{z}^{\prime}\right)$ at $z$, it holds that

$$
\begin{aligned}
& D_{z} f^{n}\left(\alpha_{z}\right) \\
= & \left(u_{f^{n}(z)}, n b u_{f^{n}(z)}+u_{f^{n}(z)}^{\prime}\right) \\
= & \left(u_{f^{n}(z)}, u_{f^{n}(z)}^{\prime}\right) B^{n} \\
= & \alpha_{f^{n}(z)} B^{n}
\end{aligned}
$$

where

$$
B=\left(\begin{array}{ll}
1 & b \\
0 & 1
\end{array}\right)
$$

At the same time we can find for any $\gamma_{z}=\left(w_{z}, w_{z}^{\prime}\right) \in \mathcal{U}_{2}^{\#}(z)$ a non-degenerate matrix

$$
C=\left(\begin{array}{ll}
c_{1} & c_{1}^{\prime} \\
c_{2} & c_{2}^{\prime}
\end{array}\right)
$$

such that $\gamma_{z}=\alpha_{z} C$. Thus

$D_{z} f^{n}\left(\gamma_{z}\right)=\alpha_{f^{n}(z)} B^{n} C=\left(\left(c_{1}+n b c_{2}\right) u_{f^{n}(z)}+c_{2} u_{f^{n}(z)}^{\prime},\left(c_{1}^{\prime}+n b c_{2}^{\prime}\right) u_{f^{n}(z)}+c_{2}^{\prime} u_{f^{n}(z)}^{\prime}\right)$.

Denote

$$
\begin{aligned}
& w_{z n}=\left(c_{1}+n b c_{2}\right) u_{f^{n}(z)}+c_{2} u_{f^{n}(z)}^{\prime} \\
& w_{z n}^{\prime}=\left(c_{1}^{\prime}+n b c_{2}^{\prime}\right) u_{f^{n}(z)}+c_{2}^{\prime} u_{f^{n}(z)}^{\prime}
\end{aligned}
$$

Therefore,

$$
\operatorname{Vol}\left(D^{\#} f^{n}\left(\gamma_{z}\right)\right)=\frac{\operatorname{Vol}\left(D f^{n}\left(\gamma_{z}\right)\right)}{\left\|w_{z n}\right\|\left\|w_{z n}^{\prime}\right\|}=\frac{\operatorname{Vol}\left(\alpha_{f^{n}(z)}\right)\left|\operatorname{det} B^{n}\right||\operatorname{det} C|}{\left\|w_{z n}\right\|\left\|w_{z n}^{\prime}\right\|}=\frac{|\operatorname{det} C|}{\left\|w_{z n}\right\|\left\|w_{z n}^{\prime}\right\|} .
$$

Since $\operatorname{det} C \neq 0, c_{2}$ and $c_{2}^{\prime}$ cannot vanish simultaneously. This means either $\left\|w_{z n}\right\| \rightarrow \infty$ or $\left\|w_{z n}^{\prime}\right\| \rightarrow \infty$ as $n \rightarrow+\infty$. Consequently, $\operatorname{Vol}\left(D^{\#} f^{n}\left(\gamma_{z}\right)\right) \rightarrow 0$ as $n \rightarrow \infty$, and then by the arbitrariness of $z$ and $\gamma_{z}$, we have $k^{*}(\Gamma)=1<2=\operatorname{dim} \mathbb{S}^{2}$. By choosing a suitable function $\phi: \mathbb{R} \rightarrow \mathbb{R}$, one constructs a diffeomorphism $f$ and a periodic orbit for $f$ on $\Gamma$. Then the atomic measure on this periodic orbit $m \in \mathcal{M}_{\text {erg }}\left(\mathbb{S}^{2}, f\right)$ satisfies

$$
k^{*}(m)=1<\operatorname{dim} \mathbb{S}^{2}
$$

Let $m \in \mathcal{M}_{\text {erg }}(M, f)$. Theorem [1.5 states that for $m-$ a.e. $x \in M$ there exists a mean linearly independent $k^{*}(m)$-frame $\alpha \in \mathcal{U}_{k^{*}(m)}^{\#}(x)$, and all these frames in $\mathcal{U}_{k^{*}(m)}^{\#}$ form a $\mu$-full measure subset for some $\mu \in \mathcal{M}_{\text {erg }}\left(\mathcal{U}_{k^{*}(m)}^{\#}\right)$ with $\pi_{*}(\mu)=m$. The next example shows that there possibly exists a $k^{*}(m)$-frame $\beta \in \mathcal{U}_{k^{*}(m)}^{\#}(x)$ that is not mean linearly independent, even if it generates the same linear subspace in $T_{x} M$ as a mean linearly independent frame generates.

Example 3. Let $m$ be an ergodic measure on $M$ with $k^{*}(m)=k(m)=\operatorname{dim} M=2$ and let $\lambda_{1}>\lambda_{2}$ be its Lyapunov exponents with different signs. Take for $m-$ a.e. $x \in M$ the invariant and orthogonal splitting $T_{x} M=E_{1}(x) \oplus E_{2}(x)$ as in the Oseledec Theorem [9]. Take and fix unit vectors $e_{1}$ in $E_{1}(x)$ and $e_{2}$ in $E_{2}(x)$. 
Then $\alpha:=\left(e_{1}, e_{2}\right)$ is mean linearly independent by Definition 1.1 and the Birkhoff Ergodic Theorem. Let

$$
\beta:=\left(\frac{e_{1}+e_{2}}{\left\|e_{1}+e_{2}\right\|}, \frac{e_{1}-e_{2}}{\left\|e_{1}-e_{2}\right\|}\right) .
$$

Then

$$
\operatorname{Vol}\left(D^{\#} f^{n} \beta\right)=\frac{\operatorname{Vol}\left(D f^{n} \beta\right)}{\left\|D f^{n} \frac{e_{1}+e_{2}}{\left\|e_{1}+e_{2}\right\|}\right\|\left\|D f^{n} \frac{e_{1}-e_{2}}{\left\|e_{1}-e_{2}\right\|}\right\|} .
$$

Since neither $e_{1}+e_{2}$ nor $e_{1}-e_{2}$ is contained in any invariant bundle, we have

$$
\lim _{n \rightarrow+\infty} \frac{1}{n} \log \left\|D f^{n} \frac{e_{1} \pm e_{2}}{\left\|e_{1} \pm e_{2}\right\|}\right\|=\lambda_{1} .
$$

On the other hand, we deduce from [15], [16] that

$$
\lim _{n \rightarrow+\infty} \frac{1}{n} \log \operatorname{Vol}\left(D f^{n} \beta\right)=\lim _{n \rightarrow+\infty} \frac{1}{n} \log \operatorname{Vol}\left(D f^{n} \alpha\right)=\lambda_{1}+\lambda_{2} .
$$

Thus

$$
\lim _{n \rightarrow+\infty} \frac{1}{n} \log \operatorname{Vol}\left(D^{\#} f^{n} \beta\right)=\lambda_{2}-\lambda_{1}<0,
$$

and hence $\operatorname{Vol}\left(D^{\#} f^{n}(\beta)\right) \rightarrow 0$ as $n \rightarrow+\infty$, which implies that $\beta$ is not mean linearly independent by Definition 1.1 .

2.3. A continuity property for $\tau$ and $\tilde{\tau}$. In this subsection we will prove the continuity of $\tau: \mathcal{U}_{\ell}^{\#} \rightarrow(0,+\infty)$ given before Definition 1.2 and $\tilde{\tau}: \mathcal{M}_{\text {erg }}\left(\mathcal{U}_{\ell}^{\#}, D^{\#} f\right) \rightarrow$ $[0,+\infty)$ given in Definition [1.6, which are necessary for proving Theorem 1.5] and Theorem 1.7.

Denote by $\mathcal{L}\left(\mathbb{R}^{\ell}\right)$ the space of all linear maps on $\mathbb{R}^{\ell}$ and by $\sigma(L)$ the set of all eigenvalues of a linear map $L \in \mathcal{L}\left(\mathbb{R}^{\ell}\right)$. For any $\epsilon>0$, set

$$
\sigma_{\epsilon}(L):=\left\{\tau \in \mathbb{C} \mid \exists \tau^{\prime} \in \sigma(L), \text { s.t. }\left|\tau-\tau^{\prime}\right|<\epsilon\right\} .
$$

Lemma 2.4 and Lemma 2.5 are cited from [10].

Lemma 2.4. For given $L \in \mathcal{L}\left(\mathbb{R}^{\ell}\right)$ and $\epsilon>0$ there is $\delta>0$ such that $\sigma(T) \subset \sigma_{\epsilon}(L)$ whenever $\|T-L\|<\delta$.

Lemma 2.5. Let $L \in \mathcal{L}\left(\mathbb{R}^{\ell}\right)$ and $\tau \in \sigma(L)$. There exist $\epsilon>0$ and $\delta>0$ with the property that for any $T \in \mathcal{L}\left(\mathbb{R}^{\ell}\right)$ with $\|T-L\|<\delta$ the cardinality of $\sigma(T) \cap B_{\epsilon}(\tau)$ is not more than the multiplicity of $\tau$, where $B_{\epsilon}(\tau)=\left\{\tau^{\prime} \in \mathbb{C}|| \tau-\tau^{\prime} \mid<\epsilon\right\}$.

Proposition 2.6. The function $\tau: \mathcal{U}_{\ell}^{\#} \rightarrow(0,+\infty)$ and $\tilde{\tau}: \mathcal{M}_{\text {erg }}\left(\mathcal{U}_{\ell}^{\#}, D^{\#} f\right) \rightarrow$ $[0,+\infty)$ are continuous.

Proof. Since $A(\alpha)$ is continuous with $\alpha$, we can define

$$
d(\alpha, \beta):=\|A(\alpha)-A(\beta)\|
$$

when $\alpha, \beta \in \mathcal{U}_{\ell}^{\#}$ are close enough. Assume that $\tau$ is not continuous at a frame $\alpha_{0} \in \mathcal{U}_{\ell}^{\#}$. Then there must exist a constant $\epsilon_{0}>0$ such that for any $\delta>0$ we can find another frame $\beta=\beta(\delta) \in \mathcal{U}_{\ell}^{\#}$ satisfying $d\left(\alpha_{0}, \beta\right)<\delta$ but $\left|\tau\left(\alpha_{0}\right)-\tau(\beta)\right|>2 \epsilon_{0}$. Arrange the eigenvalues of $A\left(\alpha_{0}\right)$ in decreasing order as $\left\{\tau_{1}>\tau_{2}>\cdots>\tau_{t}>\right.$ $\left.\tau\left(\alpha_{0}\right)\right\}, t \leq \ell-1$. By Lemma 2.5, there are real numbers $\epsilon_{i}>0, \delta_{1}>0$ and $\delta^{\prime}>0$ 
such that for any $\gamma \in \mathcal{U}_{\ell}^{\#}$ with $d\left(\gamma, \alpha_{0}\right)<\delta^{\prime}$, the following holds:

(a) the cardinality $\# \sigma(\gamma) \cap B_{\epsilon_{i}}\left(\tau_{i}\right)$ is not more than the multiplicity of $\tau_{i}$, and

(b) the cardinality $\# \sigma(\gamma) \cap B_{\delta_{1}}\left(\tau\left(\alpha_{0}\right)\right)$ is not more than the multiplicity of $\tau\left(\alpha_{0}\right)$ and

(c) $B_{\epsilon_{i}}\left(\tau_{i}\right) \cap B_{\delta_{1}}\left(\tau\left(\alpha_{0}\right)\right)=\emptyset, i=1, \ldots, t$.

Take

$$
0<\epsilon \leq \min \left\{\epsilon_{i}, \delta_{1} ; i=0,1, \ldots, t\right\} .
$$

Choose $\delta<\min \left\{\epsilon, \delta^{\prime}\right\}$ by Lemma 2.4 such that each $\gamma \in \mathcal{U}_{\ell}^{\#}$ with $d\left(\gamma, \alpha_{0}\right)<\delta$ satisfies $\tau(\gamma) \in \bigcup_{i=1}^{t} B_{\epsilon}\left(\tau_{i}\right) \cup B_{\epsilon}\left(\tau\left(\alpha_{0}\right)\right)$. From the choice of $\epsilon_{0}$ and $\beta=\beta(\delta)$ we have $\tau(\beta) \in \bigcup_{i=1}^{t} B_{\epsilon_{i}}\left(\tau_{i}\right)$. Recall that $\tau(\beta)$ is the smallest eigenvalue of $A(\beta)$ and hence there is no eigenvalue of $A(\beta)$ inside $B_{\epsilon}\left(\tau\left(\alpha_{0}\right)\right)$. So we deduce by Lemma 2.4 and Lemma 2.5 that

$$
\begin{aligned}
\operatorname{dim} M & =\# \sigma(\beta)=\#\left[\sigma(\beta) \cap B_{\epsilon}\left(\tau\left(\alpha_{0}\right)\right)\right]+\#\left[\sigma(\beta) \cap \bigcup_{i=1}^{t} B_{\epsilon}\left(\tau_{i}\right)\right] \\
& =\#\left[\sigma(\beta) \cap \bigcup_{i=1}^{t} B_{\epsilon}\left(\tau_{i}\right)\right] \\
& <\# \sigma\left(\alpha_{0}\right)=\operatorname{dim} M
\end{aligned}
$$

It's a contradiction. This implies that the function $\tau$ is continuous. Additionally, $\tau$ is a bounded function by Lemma 2.1.

Let $\mu \in \mathcal{M}_{\text {erg }}\left(\mathcal{U}_{\ell}^{\#}, D^{\#} f\right)$. By the Birkhoff Ergodic Theorem, we have

$$
\tilde{\tau}(\mu)=\lim _{n \rightarrow+\infty} \frac{1}{n} \sum_{i=0}^{n-1} \tau\left(D^{\#} f^{i} \alpha\right)=\int \tau d \mu
$$

for any $\alpha \in Q_{\mu}\left(\mathcal{U}_{\ell}^{\#}, D^{\#} f\right)$. Combining this equation with the continuity of $\tau$, one can deduce that $\tilde{\tau}$ is also continuous in the weak* topology. Hence the proposition is proved.

2.4. Proof of Theorem 1.5. In this subsection we will choose an ergodic measure on $\mathcal{U}_{k}^{\#}$, where $k$ is the style number, to cover the given ergodic measure on the state manifold and then prove Theorem 1.5 .

Theorem 2.7. Let $f: M \rightarrow M$ be a $C^{1}$ diffeomorphism preserving an ergodic measure $m \in \mathcal{M}_{\text {erg }}(M, f)$. Denote by $k$ the style number $k_{+}^{*}(m), k_{-}^{*}(m)$ or $k^{*}(m)$, respectively. Denote by $\mathcal{U}_{k}^{\#}\left(Q_{m}(M, f)\right)$ the restriction bundle of $\mathcal{U}_{k}^{\#}(M)$ on $Q_{m}(M, f)$. Then there exists a $D^{\#} f$-invariant ergodic measure $\mu \in \mathcal{M}_{\text {erg }}\left(\mathcal{U}_{k}^{\#}\left(Q_{m}(M, f)\right)\right)$ so that $\pi_{*}(\mu)=m$. Conversely, if a positive integer $\ell$ satisfies

$$
\pi_{*}^{-1}(m) \cap \mathcal{M}_{\text {erg }}\left(\mathcal{U}_{\ell}^{\#}, D^{\#} f\right) \neq \emptyset,
$$

it holds that $\ell \leq k$.

Proof. We prove the case when $k=k_{+}^{*}(m)$ only, and omit the similar proof for the other cases of $k_{-}^{*}(m)$ and $k^{*}(m)$. Denote by $F:=\operatorname{Closure}\left(Q_{m}(M, f)\right)$ the closure of $Q_{m}(M, f)$; then $F$ is an $f$-invariant closed subset of $M$. Note $\left(L_{k}^{\#}(F), D^{\#} f\right)$ is a continuous homeomorphism on a compact metric space and thus $\mathcal{M}_{\text {erg }}\left(L_{k}^{\#}, D^{\#} f\right) \neq$ $\emptyset$. Clearly, $\pi \circ D^{\#} f(\alpha)=f \circ \pi(\alpha), \quad \forall \alpha \in L_{k}^{\#}(F)$. 
Step I. Find all ergodic measures on $\left(L_{k}^{\#}(F), D^{\#} f\right)$ covering $m$.

Take and fix a point $x \in Q_{m}(M, f)$, a frame $\alpha \in \mathcal{U}_{k}^{\#}(x)$ and a positive real $\epsilon>0$ so that

$$
\varlimsup_{n \rightarrow+\infty} \frac{1}{n} \sum_{i=0}^{n-1} \chi_{\alpha \epsilon}(i)>0
$$

Define a sequence of measures $\mu_{n}$ on $L_{k}^{\#}$ by

$$
\int \phi d \mu_{n}:=\frac{1}{n} \sum_{i=0}^{n-1} \phi\left(D^{\#} f^{i} \alpha\right), \quad \forall \phi \in C^{0}\left(L_{k}^{\#}, \mathbb{R}\right) .
$$

By taking a subsequence when necessary we can assume that $\mu_{n} \rightarrow \mu_{0}$. It is standard to verify that $\mu_{0}$ is a $D^{\#} f$-invariant measure and $\mu_{0}$ covers $m$, i.e., $\pi_{*}\left(\mu_{0}\right)=m$. Set

$$
Q\left(L_{k}^{\#}, D^{\#} f\right):=\bigcup_{\nu \in \mathcal{M}_{e r g}\left(L_{k}^{\#}, D^{\#} f\right)} Q_{\nu}\left(L_{k}^{\#}, D^{\#} f\right) .
$$

Then $Q\left(L_{k}^{\#}, D^{\#} f\right)$ is a $D^{\#} f$-invariant total measure subset in $L_{k}^{\#}$. We have

$$
\begin{aligned}
& m\left(Q_{m}(M, f) \cap \pi Q\left(L_{k}^{\#}, D^{\#} f\right)\right) \\
\geq & \mu_{0}\left(\pi^{-1} Q_{m}(M, f) \cap Q\left(L_{k}^{\#}, D^{\#} f\right)\right) \\
= & 1 .
\end{aligned}
$$

Then the set

$$
\begin{aligned}
& \mathcal{A}:=\left\{\mu \in \mathcal{M}_{\text {erg }}\left(L_{k}^{\#}, D^{\#} f\right) \mid\right. \exists \gamma \in Q\left(L_{k}^{\#}, D^{\#} f\right), \pi(\gamma) \in Q_{m}(M, f), \text { s.t. } \\
& \lim _{n \rightarrow+\infty} \frac{1}{n} \sum_{i=0}^{n-1} \phi\left(D^{\#} f^{i} \gamma\right)=\lim _{n \rightarrow-\infty} \frac{1}{n} \sum_{i=0}^{n-1} \phi\left(D^{\#} f^{i} \gamma\right) \\
&\left.=\int_{L_{k}^{\#}} \phi d \mu, \quad \forall \phi \in C^{0}\left(L_{k}^{\#}, \mathbb{R}\right)\right\}
\end{aligned}
$$

is non-empty. It is clear that $\mu$ covers $m, \pi_{*}(\mu)=m$, for all $\mu \in \mathcal{A}$. Also, we claim that $\mathcal{A}$ coincides with the set of all the measures in $\mathcal{M}_{\text {erg }}\left(L_{k}^{\#}, D^{\#} f\right)$ that cover $m$. In fact, if $\mu \in \mathcal{M}_{\text {erg }}\left(L_{k}^{\#}, D^{\#} f\right)$ covers $m, \pi_{*} \mu=m$, from the fact that $\mu\left(Q_{\mu}\left(L_{k}^{\#}, D^{\#} f\right)\right)=1$, we have

$$
\begin{aligned}
& m\left(Q_{m}(M, f) \cap \pi Q_{\mu}\left(L_{k}^{\#}, D^{\#} f\right)\right) \\
\geq & \mu\left(\pi^{-1} Q_{m}(M, f) \cap Q_{\mu}\left(L_{k}^{\#}, D^{\#} f\right)\right) \\
= & 1 .
\end{aligned}
$$

Thus there is $\beta \in Q_{\mu}\left(L_{k}^{\#}, D^{\#} f\right)$ with $\pi(\beta) \in Q_{m}(M, f)$, which means $\mu \in \mathcal{A}$. Therefore,

$$
\mathcal{A}=\left\{\mu \in \mathcal{M}_{\text {erg }}\left(L_{k}^{\#}, D^{\#} f\right) \mid \pi_{*}(\mu)=m\right\} .
$$

Step II. Choose for $m$ a covering measure $\mu \in \mathcal{A}$ so that $Q_{\mu}\left(L_{k}^{\#}, D^{\#} f\right) \subset$ $\mathcal{U}_{k}^{\#}\left(Q_{m}(M, f)\right)$.

When $\mu \in \mathcal{M}_{\text {erg }}\left(L_{k}^{\#}, D^{\#} f\right) \backslash \mathcal{A}$, then $\pi_{*}(\mu) \neq m$ by Step I, and thus

$$
\pi^{-1} Q_{m}(M, f) \cap Q_{\mu}\left(L_{k}^{\#}, D^{\#} f\right)=\emptyset .
$$


For the reference measure $\mu_{0}$ defined in Step I, it follows that

$$
\begin{aligned}
\mu_{0} & \left(\bigcup_{\mu \in \mathcal{A}}\left(Q_{\mu}\left(L_{k}^{\#}, D^{\#} f\right) \cap F_{\mu}\right)\right. \\
& =\mu_{0}\left(\pi^{-1} Q_{m}(M, f) \cap\left(\bigcup_{\mu \in \mathcal{A}}\left(Q_{\mu}\left(L_{k}^{\#}, D^{\#} f\right) \cap F_{\mu}\right)\right)\right) \\
& =\mu_{0}\left(\pi^{-1} Q_{m}(M, f) \cap\left(\bigcup_{\mu \in \mathcal{M}_{\text {erg }}\left(L_{k}^{\#}, D^{\#} f\right)}\left(Q_{\mu}\left(L_{k}^{\#}, D^{\#} f\right) \cap F_{\mu}\right)\right)\right) \\
& =1
\end{aligned}
$$

where $F_{\mu}$ is a $\mu$-full measure set, $\mu \in \mathcal{A}$.

We claim that

$$
\mu_{0}\left(\mathcal{U}_{k}^{\#}(F) \cap\left(\bigcup_{\mu \in \mathcal{A}}\left(Q_{\mu}\left(L_{k}^{\#}, D^{\#} f\right) \cap F_{\mu}\right)\right)\right)>0
$$

for any $\mu$-full measure set $F_{\mu}$, where $\mu \in \mathcal{A}$.

Let us recall the chosen frame $\alpha \in \mathcal{U}_{k}^{\#}(x)$, where $x \in Q_{m}(M, f)$, and the chosen real $\epsilon>0$ as in (2.1). We define a continuous function $\phi: L_{k}^{\#} \rightarrow \mathbb{R}$ with $0 \leq \phi \leq 1$ so that $\phi\left\{\beta: \operatorname{Vol}(\beta) \leq \frac{3 \epsilon}{4}\right\}=0$ and $\phi\{\beta: \epsilon \leq \operatorname{Vol}(\beta) \leq 1\}=1$ by Urysohn's Lemma. Denote by $\chi_{\left\{\beta: \frac{\epsilon}{2} \leq \operatorname{Vol}(\beta) \leq 1\right\}}$ the character function on $\left\{\beta \in L_{k}^{\#}: \frac{\epsilon}{2} \leq\right.$ $\operatorname{Vol}(\beta) \leq 1\}$. Then

$$
\phi \leq \chi_{\left\{\beta: \frac{\epsilon}{2} \leq \operatorname{Vol}(\beta) \leq 1\right\}} .
$$

Note that the sequence of measures $\left\{\mu_{n}\right\}$ defined below (2.1) converge to $\mu_{0}$. It holds that

$$
\begin{aligned}
\mu_{n}\left\{\beta: \frac{\epsilon}{2} \leq \operatorname{Vol}(\beta) \leq 1\right\} & =\int \chi_{\left\{\beta: \frac{\epsilon}{2} \leq \operatorname{Vol}(\beta) \leq 1\right\}} d \mu_{n} \\
\geq \int \phi d \mu_{n} & =\frac{1}{n} \sum_{i=0}^{n-1} \phi\left(D^{\#} f^{i} \alpha\right) \\
& \geq \frac{1}{n} \sum_{i=0}^{n-1} \chi_{\alpha \epsilon}(i) .
\end{aligned}
$$

This shows by $(2.1)$ that $\mu_{n}\left\{\beta: \frac{\epsilon}{2} \leq \operatorname{Vol}(\beta) \leq 1\right\}>0$ for $n$ large enough. Since $\left\{\beta: \frac{\epsilon}{2} \leq \operatorname{Vol}(\beta) \leq 1\right\}$ is a closed subset in $L_{k}^{\#}$ and $\mu_{n} \rightarrow \mu_{0}$ as $n \rightarrow+\infty$, we have

$$
\mu_{0}\left\{\beta: \frac{\epsilon}{2} \leq \operatorname{Vol}(\beta) \leq 1\right\} \geq \overline{\lim }_{n \rightarrow+\infty} \mu_{n}\left\{\beta: \frac{\epsilon}{2} \leq \operatorname{Vol}(\beta) \leq 1\right\}>0 .
$$

So $\mu_{0}\left(\mathcal{U}_{k}^{\#}(F)\right) \geq \mu_{0}\left\{\beta \in L_{k}^{\#}: \quad \frac{\epsilon}{2} \leq \operatorname{Vol}(\alpha) \leq 1\right\}>0$. This together with (2.2) implies (2.3).

Now we assert that there is a measure $\mu^{*} \in \mathcal{A}$ with

$$
\mu^{*}\left(\mathcal{U}_{k}^{\#}(F) \cap E_{\mu^{*}}\right)>0
$$

for some $f$-invariant and $\mu^{*}$-full measure set $E_{\mu^{*}}$. Otherwise, we have for any $\mu \in \mathcal{A}$ and any $f$-invariant and $\mu$-full measure set $E_{\mu}$,

$$
\mu\left(\mathcal{V}_{k}^{\#}(F) \cap E_{\mu}\right)=1,
$$

and thus, by taking $E_{\mu}$ as $Q_{\mu}$, the basin of $\mu$, it holds that

$$
\mu\left(\mathcal{V}_{k}^{\#}(F) \cap Q_{\mu}\right)=1 .
$$


Set $F_{\mu}=\mathcal{V}_{k}^{\#}(F) \cap Q_{\mu}$; then it is $D^{\#} f$-invariant and of $\mu$-full measure. Then

$$
Q_{\mu} \cap F_{\mu}=F_{\mu} \subset \mathcal{V}_{k}^{\#}(F),
$$

i.e.,

$$
Q_{\mu} \cap F_{\mu} \cap \mathcal{U}_{k}^{\#}(F)=\emptyset .
$$

This contradicts (2.3) and thus proves the assertion. Further, the ergodicity of $\mu^{*}$ implies that

$$
Q_{\mu^{*}}\left(L_{k}^{\#}, D^{\#} f\right) \cap E_{\mu^{*}} \subset \mathcal{U}_{k}^{\#}(F) .
$$

Note that since $\pi Q_{\mu^{*}}\left(L_{k}^{\#}, D^{\#} f\right) \subset Q_{m}(M, f)$, we obtain

$$
Q_{\mu^{*}}\left(L_{k}^{\#}, D^{\#} f\right) \cap E_{\mu^{*}} \subset \mathcal{U}_{k}^{\#}\left(Q_{m}(M, f)\right) .
$$

So $\mu^{*} \in \mathcal{M}_{\text {erg }}\left(\mathcal{U}_{k}^{\#}\left(Q_{m}(M, f)\right)\right)$. Without any confusion, we write $\mu^{*}$ as $\mu$.

Step III. The maximum property of the style number.

Assume that there exists an ergodic measure $\mu$ in $\mathcal{M}_{\text {erg }}\left(\mathcal{U}_{\ell}^{\#}, D^{\#} f\right)$ covering $m$. For any frame $\alpha$ in $Q_{\mu}$, since the function $\tau: \mathcal{U}_{\ell}^{\#} \rightarrow \mathbb{R}$ is continuous by Proposition 2.6. we have that

By Lemma 2.1,

$$
\varlimsup_{n \rightarrow+\infty} \frac{1}{n} \sum_{i=0}^{n-1} \tau\left(D^{\#} f^{i} \alpha\right)=\int_{Q_{\mu}} \tau d \mu .
$$

$$
\int_{Q_{\mu}} \tau d \mu \geq \int_{Q_{\mu}} \frac{\operatorname{det}(A(\alpha))}{\ell^{\ell-1}} d \mu=\frac{1}{\ell^{\ell-1}} \int_{Q_{\mu}} \operatorname{det}(A(\alpha)) d \mu>0 .
$$

So

$$
\varlimsup_{n \rightarrow+\infty} \frac{1}{n} \sum_{i=0}^{n-1} \tau\left(D^{\#} f^{i} \alpha\right)>0 .
$$

According to Proposition 2.3 and Definition 1.3 , we can deduce that $\ell \leq k$.

Remark. In [2], Dai obtained similar results as the first part of Theorem 2.7 for $C^{1}$ vector fields.

Proof of Theorem 1.5. Let $k=k_{+}^{*}(m)$. We recall that $F=\operatorname{Closure}\left(Q_{m}(M, f)\right)$. Then $L_{k}^{\#}(F)$ is compact and $D^{\#} f$-invariant. Take and fix

$$
\mu \in \mathcal{M}_{\text {erg }}\left(\mathcal{U}_{k}^{\#}\left(Q_{m}(M, f)\right)\right)
$$

as in Theorem 2.7 to cover $m$. Then $\pi Q_{\mu}\left(L_{k}^{\#}, D^{\#} f\right) \subset Q_{m}(M, f)$ and $m\left(\pi Q_{\mu}\left(L^{\#}(F), D^{\#} f\right)\right)=1$.

Now we prove Theorem 1.5)(1)(2). The proof is an adaption of the proof to the main theorem about style number in [6].

For every positive integer $j$ we set $U_{j}:=\left\{\gamma \in L_{k}^{\#}(F) \mid \operatorname{Vol}(\gamma)>\frac{1}{j}\right\}$ and $W_{j}:=$ $\left\{\gamma \in L_{k}^{\#}(F) \mid \operatorname{Vol}(\gamma) \geq \frac{1}{j}\right\}$. Then

$$
U_{1} \subset W_{1} \subset \cdots \subset U_{j} \subset W_{j} \subset U_{j+1} \subset \cdots .
$$

Take by Urysohn's Lemma a continuous function $\phi_{j}: L_{k}^{\#} \rightarrow \mathbb{R}$ with $0 \leq \phi_{j} \leq 1$ so that $\phi_{j}\left(W_{j}\right)=1$ and $\phi_{j}\left(L_{k}^{\#} \backslash U_{j+1}\right)=0$. Then

$$
\chi_{W_{j}} \leq \phi_{j} \leq \chi_{W_{j+1}},
$$

where $\chi_{W_{j}}$ and $\chi_{W_{j+1}}$ denote the character functions for $W_{j}$ and $W_{j+1}$, respectively. 
For each $x \in \pi Q_{\mu}\left(L_{k}^{\#}, D^{\#} f\right)$ take $\alpha \in Q_{\mu}\left(\mathcal{U}_{k}^{\#}, D^{\#} f\right)$ with $\pi(\alpha)=x$. Then we have

$$
\begin{aligned}
& \lim _{n \rightarrow+\infty} \frac{1}{n} \Sigma_{i=0}^{n-1} \chi_{\alpha \frac{1}{j+1}}\left(D^{\#} f^{i}\right) \\
& \quad=\lim _{n \rightarrow+\infty} \frac{1}{n} \sum_{i=0}^{n-1} \chi_{W_{j+1}}\left(D^{\#} f^{i}(\alpha)\right) \\
& \geq \lim _{n \rightarrow+\infty} \frac{1}{n} \Sigma_{i=0}^{n-1} \phi_{j}\left(D^{\#} f^{i} \alpha\right) \\
& \quad=\int \phi_{j} d \mu \\
& \geq \int \chi_{W_{j}} d \mu \\
& \quad=\mu\left(W_{j}\right) \rightarrow 1, \quad j \rightarrow+\infty
\end{aligned}
$$

This implies that $k_{+}^{*}(x)=k_{+}^{*}(m)=k$ for $m-$ a.e. $x \in M$. A similar argument shows

$$
\lim _{n \rightarrow-\infty} \frac{1}{|n|} \sum_{i=0}^{n-1} \chi_{\alpha \frac{1}{j+1}}\left(D^{\#} f^{i}\right) \geq \mu\left(W_{j}\right) \rightarrow 1, \quad j \rightarrow+\infty .
$$

By Definition 1.2, it holds that $k_{-}^{*}(m) \geq k_{+}^{*}(m)$.

Similarly for the case $k=k_{-}^{*}(m)$, we can obtain that $k_{-}^{*}(x)=k_{-}^{*}(m)$ for $m-$ a.e. $x \in M$ and $k_{+}^{*}(m) \geq k_{-}^{*}(m)$. Consequently, for $m-$ a.e. $x \in M, k_{-}^{*}(x)=$ $k_{-}^{*}(m), \quad k_{+}^{*}(x)=k_{+}^{*}(m)$ and $k_{-}^{*}(m)=k_{+}^{*}(m)=k^{*}(m)$. Moreover, for $m-$ a.e. $x \in$ $M$, there is $\alpha \in \mathcal{U}_{k}^{\#}(x)$ satisfying (1.1), i.e.,

$$
\lim _{\epsilon \rightarrow 0} \lim _{n \rightarrow+\infty} \frac{1}{n} \Sigma_{i=0}^{n-1} \chi_{\alpha \epsilon}\left(D^{\#} f^{i}\right)=\lim _{\epsilon \rightarrow 0} \lim _{n \rightarrow-\infty} \frac{1}{|n|} \sum_{i=0}^{n-1} \chi_{\alpha \epsilon}\left(D^{\#} f^{i}\right)=1 .
$$

This proves (1)(2). This also means that every $\alpha \in Q_{\mu}\left(L_{k}^{\#}, D^{\#} f\right)$ satisfies (1.1) and $Q_{\mu}\left(L_{k}^{\#}, D^{\#} f\right)$ is a $\mu$-full measure set in $\mathcal{U}_{k}^{\#}$. Hence we proved Theorem $1.5(3)$.

\subsection{Proof of Theorem 1.7 .}

\section{Proof of Theorem 1.7 .}

Step I. Find an ergodic measure $m \in \mathcal{M}_{\text {erg }}(M, f)$ so that $k^{*}(m)=k^{*}(M)$.

Let $k:=k^{*}(M)$. We consider the topological system $\left(L_{k}^{\#}(M), D^{\#} f\right)$. Denote by $\mathcal{M}_{\text {inv }}\left(L_{k}^{\#}, D^{\#} f\right)$ the set of all $D^{\#} f$-invariant measures. By Definition 1.3. there are a $k$-frame $\alpha \in \mathcal{U}_{k}^{\#}$, a real $\epsilon>0$ and a subsequence $\left\{n_{j}\right\}_{j=0}^{+\infty}$ of positive integers such that

$$
\lim _{j \rightarrow+\infty} \frac{1}{n_{j}} \Sigma_{i=0}^{n_{j}-1} \chi_{\alpha \epsilon}(i)=\varlimsup_{n \rightarrow+\infty} \frac{1}{n} \sum_{i=0}^{n-1} \chi_{\alpha \epsilon}(i)>0 .
$$

Then it follows that

$$
\begin{aligned}
& \lim _{j \rightarrow+\infty} \frac{1}{n_{j}} \Sigma_{i=0}^{n_{j}-1} \operatorname{Vol}\left(D^{\#} f^{i} \alpha\right) \\
& \quad \geq \lim _{j \rightarrow+\infty} \frac{1}{n_{j}} \Sigma_{i=0}^{n_{j}-1} \operatorname{Vol}\left(D^{\#} f^{i} \alpha\right) \chi_{\alpha \epsilon}\left(D^{\#} f^{i}\right) \\
& \quad \geq \lim _{j \rightarrow+\infty} \frac{1}{n_{j}} \Sigma_{i=0}^{n_{j}-1} \epsilon \chi_{\alpha \epsilon}(i)>0
\end{aligned}
$$


Construct a sequence of measures $\mu_{n_{j}}$ satisfying

$$
\int \phi d \mu_{n_{j}}:=\frac{1}{n_{j}} \Sigma_{i=0}^{n_{j}-1} \phi\left(D^{\#} f^{i} \alpha\right), \quad \forall \phi \in C^{0}\left(L_{k}^{\#}, \mathbb{R}\right) .
$$

By taking a subsequence when necessary, we can assume that $\left\{\mu_{n_{j}}\right\}$ converge to some measure

$$
\tilde{\mu} \in \mathcal{M}_{i n v}\left(L_{k}^{\#}, D^{\#} f\right)
$$

It follows by (2.4) that

$$
\begin{aligned}
& \int_{L_{k}^{\#}} \operatorname{Vold} \tilde{\mu} \\
& \quad=\lim _{j \rightarrow+\infty} \int_{L_{k}^{\#}} \operatorname{Vol} d \mu_{n_{j}} \\
& \quad=\lim _{j \rightarrow+\infty} \frac{1}{n_{j}} \sum_{i=0}^{n_{j}-1} \operatorname{Vol}\left(D^{\#} f^{i} \alpha\right) \\
& \quad>0 .
\end{aligned}
$$

From the Ergodic Decomposition Theorem (see [7]), there is $\mu \in \mathcal{M}_{\text {erg }}\left(L_{k}^{\#}, D^{\#} f\right.$ ) such that

$$
\int_{L_{k}^{\#}(M)} \operatorname{Vol} d \mu>0
$$

Observe $L_{k}^{\#}=\mathcal{U}_{k}^{\#} \cup \mathcal{V}_{k}^{\#}$ and $\left.V o l\right|_{\mathcal{V}_{k}^{\#}}=0$. So $\int_{\mathcal{U}_{k}^{\#}} \operatorname{Vol} d \mu>0$ and thus $\mu\left(\mathcal{U}_{k}^{\#}\right)>0$. Since $\mathcal{U}_{k}^{\#}$ is open and invariant, $\mu \in \mathcal{M}_{\text {erg }}\left(\mathcal{U}_{k}^{\#}(M), D^{\#} f\right)$. Let $m:=\pi_{*}(\mu)$. We can check as in Theorem 2.7 and Theorem 1.5 that $k^{*}(m)=k=k^{*}(M)$.

Step II. Let $k:=k^{*}(M)$. We will find an ergodic measure $\mu_{0}$ on $\mathcal{U}_{k}^{\#}$ with the largest independence number among all ergodic measures on $\mathcal{U}_{k}^{\#}$.

Define $\tilde{\tau}: \mathcal{M}_{i n v}\left(L_{k}^{\#}, D^{\#} f\right) \rightarrow \mathbb{R}$ by $\tilde{\tau}(\mu):=\int \tau d \mu$. Then the continuity of $\tau: L_{k}^{\#} \rightarrow[0,+\infty)$ proved in Proposition 2.6 ensures that $\tilde{\tau}$ is also a continuous function under the weak ${ }^{*}$ topology. Whenever $\mu \in \mathcal{M}_{\text {erg }}\left(L_{k}^{\#}, D^{\#} f\right)$, we have

$$
\int \tau d \mu=\lim _{n \rightarrow \pm \infty} \frac{1}{|n|} \Sigma_{i=1}^{n-1} \tau\left(D^{\#} f^{i} \alpha\right)=\tilde{\tau}(\alpha),
$$

$\forall \alpha \in Q_{\mu}\left(L_{k}^{\#}, D^{\#} f\right)$, and thus $\int \tau d \mu=\sup _{\alpha \in Q_{\mu}} \tilde{\tau}(\alpha)=\tilde{\tau}(\mu)$. So $\tilde{\tau}: \mathcal{M}_{i n v} \rightarrow \mathbb{R}$ is a natural extension of $\tilde{\tau}: \mathcal{M}_{\text {erg }} \rightarrow \mathbb{R}$ in Definition 1.6.

Let $\alpha \in L_{k}^{\#}(M)$. Take $\left\{n_{j}\right\}_{j=1}^{\infty}$ such that by Definition 1.6

$$
\tilde{\tau}(\alpha)=\lim _{j \rightarrow \infty} \frac{1}{n_{j}} \Sigma_{i=1}^{n_{j}-1} \tau\left(D^{\#} f^{i} \alpha\right) .
$$

By taking a subsequence we assume that the measures $\mu_{n_{j}}$ defined by

$$
\int \phi d \mu_{n_{j}}=\frac{1}{n_{j}} \Sigma_{i=1}^{n_{j}-1} \phi\left(D^{\#} f^{i} \alpha\right), \quad \phi \in C^{0}\left(L_{k}^{\#}, \mathbb{R}\right),
$$

converge to some $\mu \in \mathcal{M}_{i n v}\left(L_{k}^{\#}, D^{\#} f\right)$. Then

$$
\tilde{\tau}(\mu)=\int \tau d \mu=\lim _{j \rightarrow+\infty} \int \tau d \mu_{n_{j}}=\lim _{j \rightarrow \infty} \frac{1}{n_{j}} \Sigma_{i=1}^{n_{j}-1} \tau\left(D^{\#} f^{i} \alpha\right)=\tilde{\tau}(\alpha) .
$$


This implies by (2.5) that

$$
\sup _{\mu \in \mathcal{M}_{i n v}} \tilde{\tau}(\mu)=\sup _{\alpha \in L_{k}^{\#}} \tilde{\tau}(\alpha)
$$

Since $\tilde{\tau}: \mathcal{M}_{i n v}\left(L_{k}^{\#}, D^{\#} f\right) \rightarrow \mathbb{R}$ is continuous under the weak* topology (see Proposition 2.6) and $\mathcal{M}_{\text {inv }}\left(L_{k}^{\#}, D^{\#} f\right)$ is compact, we can take $\mu_{1} \in \mathcal{M}_{i n v}\left(L_{k}^{\#}, D^{\#} f\right)$ so that

$$
\tilde{\tau}\left(\mu_{1}\right)=\sup _{\mu \in \mathcal{M}_{i n v}} \tilde{\tau}(\mu)=\sup _{\alpha \in L_{k}^{\#}} \tilde{\tau}(\alpha) .
$$

Claim I. There is $\mu_{0} \in \mathcal{M}_{\text {erg }}\left(L_{k}^{\#}, D^{\#} f\right)$ so that

$$
\tilde{\tau}\left(\mu_{0}\right)=\tilde{\tau}\left(\mu_{1}\right) .
$$

In fact, otherwise, it could have

$$
\tilde{\tau}(\mu)<\tilde{\tau}\left(\mu_{1}\right), \quad \forall \mu \in \mathcal{M}_{\text {erg }}\left(L_{k}^{\#}, D^{\#} f\right) .
$$

Set $Q:=\bigcup_{\mu \in \mathcal{M}_{i n v}} Q_{\mu}\left(L_{k}^{\#}, D^{\#} f\right)$. Then $\mu(Q)=1$ for all $\mu \in \mathcal{M}_{i n v}\left(L_{k}^{\#}, D^{\#} f\right)$. By the Ergodic Decomposition Theorem (see Theorem 2.16 in [7]), this deduces a contradiction as follows:

$$
\begin{aligned}
\tilde{\tau}\left(\mu_{1}\right) & =\int_{L_{k}^{\#}} \tau d \mu_{1} \\
& =\int_{Q}\left(\int_{L_{k}^{\#}} \tau d \mu\right) d \mu_{1} \\
& =\int_{Q} \tilde{\tau}(\mu) d \mu_{1} \\
& <\int_{Q} \tilde{\tau}\left(\mu_{1}\right) d \mu_{1}=\tilde{\tau}\left(\mu_{1}\right) .
\end{aligned}
$$

So Claim I follows.

Take and fix $\mu_{0} \in \mathcal{M}_{\text {erg }}\left(L_{k}^{\#}, D^{\#} f\right)$ such that

$$
\tilde{\tau}\left(\mu_{0}\right)=\sup _{\alpha \in L_{k}^{\#}} \tilde{\tau}(\alpha)=\sup _{\mu \in \mathcal{M}_{\text {erg }}} \tilde{\tau}(\mu) .
$$

Claim II. $\tilde{\tau}\left(\mu_{0}\right)>0$.

Since $k^{*}(m)=k$ for some $m \in \mathcal{M}_{\text {erg }}(M, f)$ by Step I, we can take $\mu_{2} \in$ $\mathcal{M}_{\text {erg }}\left(\mathcal{U}_{k}^{\#}\right)$ with $\pi_{*}\left(\mu_{2}\right)=m$ so that (1.1) in Theorem 1.5 follows for $\mu_{2}-$ a.e. $\alpha \in$ $\mathcal{U}_{k}^{\#}$. So

$$
\lim _{n \rightarrow \infty} \frac{1}{n} \sum_{i=1}^{n-1} \chi_{\alpha \epsilon}\left(D^{\#} f^{i}\right)>0, \quad \mu_{2}-\text { a.e. } \alpha \in \mathcal{U}_{k}^{\#},
$$

for small $\epsilon>0$. By Proposition 2.3

$$
\lim _{n \rightarrow \infty} \frac{1}{n} \sum_{i=1}^{n-1} \tau\left(D^{\#} f^{i} \alpha\right)>0, \quad \mu_{2}-\text { a.e. } \alpha \in \mathcal{U}_{k}^{\#} .
$$

Since $\tau$ is continuous by Proposition 2.6 and $\mu_{2}$ is ergodic, we have by the Birkhoff Ergodic Theorem, $\tilde{\tau}\left(\mu_{2}\right)>0$. This implies $\tilde{\tau}\left(\mu_{0}\right)>0$. Claim II follows.

Take and fix $\alpha \in Q_{\mu_{0}}\left(L_{k}^{\#}, D^{\#} f\right)$. Then by Claim II

$$
0<\tilde{\tau}\left(\mu_{0}\right)=\lim _{n \rightarrow \infty} \frac{1}{n} \sum_{i=1}^{n-1} \tau\left(D^{\#} f^{i} \alpha\right)
$$


which implies by Proposition 2.3 that

$$
k^{*}\left(\pi_{*} \mu_{0}\right)=k^{*}(M) .
$$

Let $m_{0}:=\pi_{*}\left(\mu_{0}\right)$. Then $m_{0} \in \mathcal{M}_{\text {erg }}(M, f)$. The ergodic measures $m_{0}$ and $\mu_{0}$ with $\pi_{*}\left(\mu_{0}\right)=m_{0}$ meet the requirement in Theorem 1.7. This completes the proof.

\section{APPROXIMATION PROPERTY OF INVARIANT MEASURES}

3.1. Periodic shadowing. We start this subsection by introducing some preliminary concepts and lemmas. Let $f: M \rightarrow M$ be a $C^{1}$ diffeomorphism on a compact smooth Riemannian manifold $M$.

Pesin set (11, 12]). Given $\lambda, \mu \gg \varepsilon>0$, and for all $k \in \mathbb{Z}^{+}$, we define $\Lambda_{k}=$ $\Lambda_{k}(\lambda, \mu ; \varepsilon)$ to be all points $x \in M$ for which there is a splitting $T_{x} M=E^{s}(x) \oplus E^{u}(x)$ with invariant property $D_{x} f^{m}\left(E^{s}(x)\right)=E^{s}\left(f^{m} x\right)$ and $D_{x} f^{m}\left(E^{u}(x)\right)=E^{u}\left(f^{m} x\right)$ satisfying:

(a) $\left\|\left.D f^{n}\right|_{E^{s}\left(f^{m} x\right)}\right\| \leq e^{\varepsilon k} e^{-(\lambda-\varepsilon) n} e^{\varepsilon|m|}, \forall m \in \mathbb{Z}, n \geq 1$;

(b) $\left\|\left.D f^{-n}\right|_{E^{u}\left(f^{m} x\right)}\right\| \leq e^{\varepsilon k} e^{-(\mu-\varepsilon) n} e^{\varepsilon|m|}, \forall m \in \mathbb{Z}, n \geq 1$;

(c) $\tan \left(\angle\left(E^{s}\left(f^{m} x\right), E^{u}\left(f^{m} x\right)\right)\right) \geq e^{-\varepsilon k} e^{-\varepsilon|m|}, \forall m \in \mathbb{Z}$.

We set $\Lambda=\Lambda(\lambda, \mu ; \varepsilon)=\bigcup_{k=1}^{+\infty} \Lambda_{k}$ and call $\Lambda$ a Pesin set. It is obvious that if $\varepsilon_{1}<\varepsilon_{2}$, then $\Lambda\left(\lambda, \mu ; \varepsilon_{1}\right) \subseteq \Lambda\left(\lambda, \mu ; \varepsilon_{2}\right)$.

Suppose $f$ preserves an ergodic hyperbolic measure $\tilde{\omega}$. Then $\tilde{\omega}$ has $s(s \leq d=$ $\operatorname{dim} M)$ non-zero Lyapunov exponents

$$
\lambda_{1}<\cdots<\lambda_{r}<0<\lambda_{r+1}<\cdots<\lambda_{s}
$$

with an associated Oseledec splitting

$$
T_{x} M=E_{1}(x) \oplus \cdots \oplus E_{s}(x), \quad x \in O(\tilde{\omega}),
$$

where $O(\tilde{\omega})$ is the Oseledec basin of $\tilde{\omega}$. We denote by

$$
\lambda=\left|\lambda_{r}\right|, \quad \mu=\lambda_{r+1},
$$

the absolute value of the largest negative Lyapunov exponent and the smallest positive Lyapunov exponent, respectively. Let

$$
E^{s}(x)=E_{1}(x) \oplus \cdots \oplus E_{r}(x), \quad E^{u}(x)=E_{r+1}(x) \oplus \cdots \oplus E_{s}(x) .
$$

Then we obtain a Pesin set $\Lambda=\Lambda(\lambda, \mu ; \varepsilon)$ for a small $\varepsilon$. We call it the Pesin set associated with $\tilde{\omega}$. It follows (see, for example, Proposition 4.2 in [13]) that $\tilde{\omega}(\Lambda \backslash O(\tilde{\omega}))+\tilde{\omega}(O(\tilde{\omega}) \backslash \Lambda)=0$.

The following statements are elementary:

(a) $\Lambda_{1} \subseteq \Lambda_{2} \subseteq \Lambda_{3} \subseteq \cdots$;

(b) $f\left(\Lambda_{k}\right) \subseteq \Lambda_{k+1}, f^{-1}\left(\Lambda_{k}\right) \subseteq \Lambda_{k+1}$;

(c) $\Lambda_{k}$ is compact for $\forall k \geq 1$;

(d) $\forall k \geq 1$ the splitting $x \rightarrow E^{u}(x) \oplus E^{s}(x)$ depends continuously on $x \in \Lambda_{k}$.

Shadowing Lemma and Closing Lemma. Let $\left\{\delta_{k}\right\}_{k=1}^{+\infty}$ be a sequence of positive real numbers. Let $\left\{x_{n}\right\}_{n=-\infty}^{+\infty}$ be a sequence of points in $\Lambda=\Lambda(\lambda, \mu, \varepsilon)$ for which there exists a sequence $\left\{s_{n}\right\}_{n=-\infty}^{+\infty}$ of positive integers satisfying:

(a) $x_{n} \in \Lambda_{s_{n}}, \forall n \in \mathbb{Z}$;

(b) $\left|s_{n}-s_{n-1}\right| \leq 1, \forall n \in \mathbb{Z}$;

(c) $d\left(f x_{n}, x_{n+1}\right) \leq \delta_{s_{n}}, \forall n \in \mathbb{Z}$; 
then we call $\left\{x_{n}\right\}_{n=-\infty}^{+\infty} a\left\{\delta_{k}\right\}_{k=1}^{+\infty}$ pseudo-orbit. Given $\eta>0$, a point $x \in M$ is an $\eta$-shadowing point for the $\left\{\delta_{k}\right\}_{k=1}^{+\infty}$ pseudo-orbit if $d\left(f^{n} x, x_{n+1}\right) \leq \eta \varepsilon_{s_{n}}, \forall n \in \mathbb{Z}$, where $\varepsilon_{k}=\varepsilon_{0} e^{-\varepsilon k}$ and $\varepsilon_{0}$ are constants.

Lemma 3.1 (Shadowing Lemma [4, 13]). Let $f: M \rightarrow M$ be a $C^{1+\alpha}$ diffeomorphism, with a non-empty Pesin set $\Lambda=\Lambda(\lambda, \mu ; \varepsilon)$ and fixed parameters: $\lambda, \mu \gg \varepsilon>0$. For all $\eta>0$ there exists a sequence $\left\{\delta_{k}\right\}_{k=1}^{+\infty}$ such that for any $\left\{\delta_{k}\right\}_{k=1}^{+\infty}$ pseudo-orbit there exists a unique $\eta$-shadowing point.

Lemma 3.2 (Closing Lemma 4). Let $f: M \rightarrow M$ be a $C^{1+\alpha}$ diffeomorphism and let $\Lambda=\Lambda(\lambda, \mu ; \varepsilon)$ be a non-empty Pesin set. For all $k \geq 1,0<\eta<1$, there exists $\beta=\beta(k, \eta)>0$ such that: if $x, f^{p} x \in \Lambda_{k}$ and $d\left(x, f^{p} x\right)<\beta$, then there exists a hyperbolic periodic point $z \in M$, with $z=f^{p} z$ and $d(z, x)<\eta \varepsilon_{k+1}<\eta$.

We point out in the next lemma that the shadowing point in the Closing Lemma above is in the Pesin set $\Lambda=\Lambda\left(\lambda, \mu ; \varepsilon^{\prime}\right)$ for some $\varepsilon^{\prime}>\varepsilon$. A similar description of this result has appeared in [5], and thus we omit the proof.

Lemma 3.3. Let $f: M \rightarrow M$ be a $C^{1+\alpha}$ diffeomorphism preserving a hyperbolic ergodic measure $\tilde{\omega}$ and let $\Lambda=\Lambda(\lambda, \mu ; \varepsilon)$ be a non-empty Pesin set associated with $\tilde{\omega}$. Then there is a positive number $\gamma_{0}<\varepsilon$ so that for any number $0<\gamma<\gamma_{0}$, one can find a small number $0<\nu=\nu(\gamma) \leq \varepsilon$ with the following property: For any $k \geq 1,0<\eta<1$, there is $\beta=\beta(k, \eta, \nu)>0$ such that, if $x, f^{p} x \in \Lambda_{k}(\lambda, \mu ; \nu)$ and $d\left(x, f^{p} x\right)<\beta$, then there exists a hyperbolic periodic point $z \in \Lambda(\lambda, \mu ; \nu+\gamma) \subset$ $\Lambda(\lambda, \mu ; \varepsilon)$, with $z=f^{p} z$ and $d(z, x)<\eta \varepsilon_{k+1}<\eta$.

The following theorem and its corollary are about how to shadow finitely many orbit segments whose lengths are bounded or whose endpoints are limited into a given Pesin block, respectively, by a periodic orbit.

Theorem 3.4. Let $f: M \rightarrow M$ be a $C^{1+\alpha}$ diffeomorphism of a compact manifold and $\tilde{\omega}$ be an ergodic hyperbolic measure with an associated Pesin set $\Lambda=\Lambda(\lambda, \mu ; \varepsilon)$. Then there exists a positive number $\nu<\frac{\varepsilon}{2}$ with the following property: For any real $\eta>0$, any integer $q \geq 1$, any sequence of points $x_{1}, \ldots, x_{\ell} \in \Lambda(\lambda, \mu ; \nu)$ and any two sequences of integers $a_{1} \leq b_{1}, \ldots, a_{\ell} \leq b_{\ell}$ with $0 \leq b_{i}-a_{i} \leq q$, there exist two integers $X \geq 1$ and $p \geq \Sigma_{i=1}^{\ell}\left(b_{i}-a_{i}\right)+\ell X$, a hyperbolic periodic point $z \in \Lambda$ and a sequence of integers $c_{1}, \ldots, c_{\ell}$ such that $f^{p} z=z$ and

$$
d\left(f^{c_{j}+i} z, f^{i} x_{j}\right)<\eta, \quad a_{j} \leq i \leq b_{j}, \quad j=1, \ldots, \ell .
$$

Proof. Take $\delta>0$ so that

$$
d(x, y)<\delta \text { implies } d\left(f^{i} x, f^{i} y\right)<\frac{\eta}{2}
$$

for any $x, y \in M$, any $i=1, \ldots, q$. For $\varepsilon$, take $\gamma_{0}>0$ and for $0<\gamma<\gamma_{0}$, take $\nu=\nu(\gamma)>0$ by Lemma 3.3. For any sequence of points

$$
x_{1}, \ldots, x_{\ell} \in \Lambda(\lambda, \mu ; \nu)
$$

take by Lemma 3.1 a sequence $\left\{\delta_{k}\right\}_{k=1}^{\infty}$ so that each $\left\{\delta_{k}\right\}_{k=1}^{\infty}$ pseudo-orbit in the Pesin set $\Lambda(\lambda, \mu ; \nu)$ can be $\frac{\eta}{2}$-traced. Let $S=\bigcup_{i=1}^{\ell}\left\{x_{i}, f\left(x_{i}\right), \ldots, f^{b_{i}-a_{i}}\left(x_{i}\right)\right\}$ and

$$
k_{0}=\min \left\{k \geq 1 \mid S \subset \Lambda_{k}(\lambda, \mu ; \nu)\right\} .
$$

Now we will consider $\Lambda_{k_{0}}(\lambda, \mu ; \nu)$. For simplicity, denote $\Lambda_{k_{0}}=\Lambda_{k_{0}}(\lambda, \mu ; \nu)$. 
Take and fix for $\Lambda_{k_{0}}$ a finite open cover $\alpha=\left\{U_{1}, \ldots, U_{r}\right\}$ by open balls $U_{i}$ in $M$ with $\operatorname{diam} U_{i}<\delta_{k_{0}}$ and $\tilde{\omega}\left(U_{i} \cap \Lambda_{k_{0}}\right)>0, \quad i=1, \ldots, r$. Since $\tilde{\omega}$ is ergodic, there exist positive integers $X_{i j}$ such that

$$
\tilde{\omega}\left[f^{-X_{i j}}\left(U_{i} \cap \Lambda_{k_{0}}\right) \cap\left(U_{j} \cap \Lambda_{k_{0}}\right)\right]>0 .
$$

Denote without confusion by $X_{i j}$ the least positive integer satisfying (3.2).

Choose two open balls $U_{i_{0}}, U_{i_{1}} \in \alpha$ with the property that $f^{a_{i}} x_{i} \in U_{i_{0}}, f^{b_{i}} x_{i} \in$ $U_{i_{1}}, i=1,2, \ldots, \ell$. By (3.2) take $y_{i} \in U_{i_{1}} \cap \Lambda_{k_{0}}$ so that $f^{X_{(i+1)_{0} i_{1}}} y_{i} \in U_{(i+1)_{0}} \cap \Lambda_{k_{0}}$ for $i=1, \ldots, \ell-1$. Take $y_{\ell} \in U_{\ell_{1}} \cap \Lambda_{k_{0}}$ so that $f^{X_{1_{0} \ell_{1}}} y_{\ell} \in U_{1_{0}} \cap \Lambda_{k_{0}}$. Hence we obtain a $\left\{\delta_{k}\right\}_{k=1}^{\infty}$ periodic pseudo-orbit in the Pesin set $\Lambda(\lambda, \mu ; \nu)$ by repeating the following finite sequence infinitely many times:

$$
\begin{aligned}
f^{a_{1}} x_{1}, \ldots, f^{b_{1}} x_{1}, f y_{1}, \ldots, f^{X_{2_{0} 1_{1}-1}} y_{1}, f^{a_{2}} x_{2}, \ldots, f^{b_{2}} x_{2}, f y_{2}, \ldots, \\
\\
f^{X_{3_{0} 2_{1}-1}} y_{2}, \ldots, f^{a_{\ell}} x_{\ell}, \ldots, f^{b_{\ell}} x_{\ell}, f y_{\ell}, \ldots, f^{X_{1_{0} \ell_{1}-1}} y_{\ell} .
\end{aligned}
$$

Let

$$
X=\min _{1 \leq i \neq j \leq r}\left\{X_{i j}\right\} .
$$

The length of the finite sequence (3.3) is clearly larger than or equal to $\Sigma_{i=1}^{\ell}\left(b_{i}-a_{i}\right)+\ell X$. By Lemma 3.1 and Lemma 3.3 there exists a unique periodic point $z \in \Lambda, \frac{\eta}{2}$-tracing the pseudo-orbit, with period $p>\Sigma_{i=1}^{\ell}\left(b_{i}-a_{i}\right)+\ell X$. This implies the existence of $c_{1}, \ldots, c_{\ell}$ which satisfy

$$
d\left(f^{c_{j}+i} z, f^{i} x_{j}\right)<\frac{\eta}{2}, \quad a_{j} \leq i \leq b_{j}, \quad j=1, \ldots, \ell .
$$

This together with (3.1) completes the proof.

Corollary 3.5. Let $f: M \rightarrow M$ be a $C^{1+\alpha}$ diffeomorphism of a compact manifold. Let $\tilde{\omega}$ be an ergodic hyperbolic measure with an associated Pesin set

$$
\Lambda=\Lambda(\lambda, \mu ; \varepsilon) .
$$

Then there exists a positive number $\nu<\frac{\varepsilon}{2}$ with the following property: Given a real $\eta>0$ and an integer $\ell \geq 1$ with $\tilde{\omega}\left(\Lambda_{\ell}(\lambda, \mu ; \nu)\right)>0$, there is an integer $X_{\ell}=X_{\ell}(\eta) \geq 1$ such that for any finitely many points

$$
x_{1}, \ldots, x_{k} \in \Lambda_{\ell}(\lambda, \mu ; \nu)
$$

and any positive integers $n_{1}, \ldots, n_{k}$, with $f^{n_{i}}\left(x_{i}\right) \in \Lambda_{\ell}(\lambda, \mu ; \nu), i=1, \ldots, k$, there exist a hyperbolic periodic point $z \in \Lambda(\lambda, \mu ; \varepsilon)$, an integer $p \geq \sum_{j=1}^{k} n_{j}+k X_{\ell}$ and a sequence of integers $c_{1}, \ldots, c_{k}$, such that $f^{p}(z)=z$, and $d\left(f^{c_{j}+i}(z), f^{i} x_{j}\right)<\eta, 1 \leq$ $i \leq n_{j}, 1 \leq j \leq k$.

Proof. For $\varepsilon>0$, take $\gamma_{0}>0$ and then take $\nu=\nu\left(\gamma_{0}\right)>0$ by Lemma 3.3. For the given real $\eta>0$, take a sequence $\left\{\delta_{k}\right\}_{k=1}^{+\infty}$ by Lemma 3.1 so that each $\left\{\delta_{k}\right\}_{k=1}^{+\infty}$ pseudo-orbit in the Pesin set $\Lambda(\lambda, \mu ; \nu)$ can be $\frac{\eta}{2}$-traced by a real orbit. For $\ell$ given in the assumption, fix $\delta_{\ell}$. We replace $\delta_{k_{0}}$ by $\delta_{\ell}$ and $\Lambda(\lambda, \mu ; \varepsilon)$ by $\Lambda(\lambda, \mu ; \nu)$ in the middle part of the proof of Theorem 3.4 and then a similar argument works; hence the conclusion holds.

For simplicity, we will identify the smaller Pesin set $\Lambda(\lambda, \mu ; \nu)$ with the one $\Lambda(\lambda, \mu ; \varepsilon)$ in the following proof. 
3.2. Density of atomic measures. Sigmund 14 proved that for uniformly hyperbolic diffeomorphisms with specification property, each invariant measure can be approximated by atomic measures. Hirayama 3 proved that each invariant measure supported by the closure of a Pesin set of a topologically mixing measure is approximated by atomic measures. Our result, Theorem 1.8 to be proved in this subsection, is to improve [3] by weakening the assumption of mixing measure to that of ergodic measure by applying Theorem 3.4 and Corollary 3.5 and the quantitative Poincaré Recurrence Lemma (i.e., Lemma 3.6). Moreover, the last inequality in Hirayama's proof in $[\underline{3}$ is not clear to us, since there is no estimation of

$$
\left|\frac{1}{s} \sum_{j=1}^{k} s_{j}\left(\frac{1}{n_{j}} \sum_{i=0}^{n_{j}-1} \xi\left(f^{i}\left(x_{j}\right)\right)\right)-\frac{1}{\# I} \sum_{i \in I} \xi\left(f^{i}(z)\right)\right|
$$

(see (3.1) and (3.3) in [3 for the notations), which is necessary to complete the proof. We will give an explicit estimation in Theorem 1.8.

Lemma 3.6. Let $f: X \rightarrow X$ be a homeomorphism of a compact metric space preserving an ergodic measure $\tilde{\omega}$. Let $\Gamma \subset X$ be a measurable set with $\tilde{\omega}(\Gamma)>0$. Take $\gamma>0$. Then there exists a measurable function $N(\Gamma, \cdot): X \rightarrow \mathbb{N}$ such that for $\tilde{\omega}-$ a.e. $x \in X$, every $n \geq N(\Gamma, x)$ and every $t \in[0,1]$, there is some $\ell \in\{0,1, \ldots, n\}$ such that $f^{\ell}(x) \in \Gamma$ and $\left|\frac{\ell}{n}-t\right|<\gamma$.

Proof. This is Lemma 3.12 in Bochi [1]. (3.4).

By using Lemma 3.6, we establish a lemma which will be applied to estimate

Lemma 3.7. Let $f: X \rightarrow X$ be a homeomorphism of a compact metric space preserving an ergodic measure $\tilde{\omega}$. Let $\Gamma_{j} \subset X$ be measurable sets with $\tilde{\omega}\left(\Gamma_{j}\right)>0$, and for $x \in \Gamma_{j}$ let

$$
S\left(x, \Gamma_{j}\right):=\left\{r \in \mathbb{N} \mid f^{r} x \in \Gamma_{j}\right\},
$$

$j=1, \ldots, k$. Take $1>\gamma>0, T \geq 1$. Then for $\tilde{\omega}-a . e . x_{j} \in \Gamma_{j}$ there exists $n_{j}=n_{j}\left(x_{j}\right) \in S\left(x_{j}, \Gamma_{j}\right)$ such that $n_{j} \geq T$ and

$$
0<\frac{\left|n_{1}-n_{j}\right|+\cdots+\left|n_{j-1}-n_{j}\right|+\left|n_{j+1}-n_{j}\right|+\cdots+\left|n_{k}-n_{j}\right|}{\sum_{j=1}^{k} n_{j}}<\gamma,
$$

where $j=1, \ldots, k$.

Proof. We prove the case when $k=2$ and $j=1$ and leave the others to the readers. Take $k_{0}>1$ with $\frac{1}{k_{0}} \leq \gamma \leq \frac{1}{k_{0}-1}$. Take measurable functions $N\left(\Gamma_{i}, \cdot\right): \Gamma_{i} \rightarrow \mathbb{N}$ as in Lemma 3.1, that is, for $\tilde{\omega}$-a.e. $x_{i} \in \Gamma_{i}$ and every $m_{i} \geq N\left(\Gamma_{i}, x_{i}\right)$ and every $t \in[0,1]$ there is some $\ell_{i} \in\left\{0,1, \ldots, m_{i}\right\} \cap S\left(x_{i}, \Gamma_{i}\right)$ such that $\left|\frac{\ell_{i}}{m_{i}}-t\right|<\gamma, i=1,2$.

Take and fix a point $x_{i} \in \Gamma_{i}$, which returns to $\Gamma_{i}$ under positive iterations infinitely many times. Let us take and fix $m_{2} \in S\left(x_{2}, \Gamma_{2}\right)$ so that

$$
m_{2}>2 \max \left\{\frac{1}{1-\gamma}(T+1), N\left(\Gamma_{1}, x_{1}\right), N\left(\Gamma_{2}, x_{2}\right), \frac{1-\gamma}{\gamma}\right\} .
$$

Denote by $[a]$ the largest integer not exceeding $a$. It holds that

$$
\left[\frac{k_{0}-1}{k_{0}} m_{2}\right]>\max \left\{\frac{1}{1-\gamma}(T+1), N\left(\Gamma_{1}, x_{1}\right), N\left(\Gamma_{2}, x_{2}\right), \frac{1-\gamma}{\gamma}\right\} .
$$


Using Lemma 3.1 by taking $t=1$ and $m_{1}=\left[\frac{k_{0}-1}{k_{0}} m_{2}\right]$, there exists $\ell_{1} \in$ $\left\{0,1, \ldots,\left[\frac{k_{0}-1}{k_{0}} m_{2}\right]\right\} \cap S\left(x_{1}, \Gamma_{1}\right)$ so that

$$
-\gamma<\frac{\ell_{1}}{\left[\frac{k_{0}-1}{k_{0}} m_{2}\right]}-1<\gamma
$$

So

$$
\left[\frac{k_{0}-1}{k_{0}} m_{2}\right](1-\gamma)<\ell_{1}<\left[\frac{k_{0}-1}{k_{0}} m_{2}\right](1+\gamma),
$$

and hence $\ell_{1}>T$. Then we have

$$
\begin{aligned}
0 & <\frac{m_{2}-\ell_{1}}{\ell_{1}+m_{2}}<\frac{m_{2}-\ell_{1}}{m_{2}}<1-\frac{\left[\frac{k_{0}-1}{k_{0}} m_{2}\right]}{m_{2}}(1-\gamma) \\
& <1-\frac{\frac{k_{0}-1}{k_{0}} m_{2}-1}{m_{2}}(1-\gamma)<\gamma+\frac{1-\gamma}{m_{2}}<2 \gamma .
\end{aligned}
$$

Replacing $2 \gamma$ by $\gamma$ and denoting $\ell_{1}$ by $n_{1}$ and $m_{2}$ by $n_{2}$, we obtain that

$$
n_{1}, n_{2}>T \quad \text { and } \quad 0<\frac{n_{2}-n_{1}}{n_{1}+n_{2}}<\gamma .
$$

This completes the proof.

Proof of Theorem 1.8, Let $\Lambda=\bigcup_{\ell=1}^{\infty} \Lambda_{\ell}$ be the Pesin set associated with $\tilde{\omega}$. We denote by $\left.\tilde{\omega}\right|_{\Lambda_{\ell}}$ the conditional measure of $\tilde{\omega}$ on $\Lambda_{\ell}$. Set $\tilde{\Lambda}_{\ell}=\operatorname{supp}\left(\left.\tilde{\omega}\right|_{\Lambda_{\ell}}\right)$ and $\tilde{\Lambda}=\bigcup_{\ell=1}^{\infty} \tilde{\Lambda}_{\ell}$. Clearly, $f^{ \pm 1} \tilde{\Lambda}_{\ell} \subset \tilde{\Lambda}_{\ell+1}$, and the sub-bundles $E^{s}(x), E^{u}(x)$ depend continuously on $x \in \tilde{\Lambda}_{\ell}$. Moreover, $\tilde{\Lambda}$ is $f$-invariant with $\tilde{\omega}$-full measure. We will show that $\tilde{\Lambda}$ meets the conditions of our theorem.

Take and fix a real $\epsilon>0$, an $f$-invariant measure $\omega \in \mathcal{M}_{\text {inv }}(f)$ with $\operatorname{supp}(\omega) \subset$ $\tilde{\Lambda}$ and a finite set $F$ of continuous functions, i.e., $F \subset C^{0}(M, \mathbb{R})$. We assume without loss of generality that $|\xi| \leq 1, \quad \forall \xi \in F$. Take $0<\eta<\frac{\epsilon}{4}$ so that

$$
d(x, y)<\eta \text { implies }|\xi(x)-\xi(y)|<\frac{\epsilon}{8}, \quad \forall x, y \in M, \forall \xi \in F .
$$

Let

$Q(f)=\left\{x \in M \mid\right.$ the limit exists $\left.\lim _{n \rightarrow+\infty} \frac{1}{n} \sum_{i=0}^{n-1} \xi\left(f^{i} x\right)=\xi^{*}(x), \quad \forall \xi \in C^{0}(M, \mathbb{R})\right\}$.

By the Birkhoff Ergodic Theorem, $\omega(Q(f))=1$ and $\tilde{\omega}(Q(f))=1$. For $x \in Q(f)$ there exists $N=N(x)$ such that

$$
\left|\frac{1}{n} \sum_{i=0}^{n-1} \xi\left(f^{i} x\right)-\xi^{*}(x)\right|<\frac{\epsilon}{8},
$$

whenever $n \geq N(x)$. We divide the following proof into several steps.

Step I. Constructing a partition of $Q(f)$.

Let $A=\sup \left\{\left|\xi^{*}(x)\right| ; x \in Q(f), \xi \in F\right\}$. Recall that by $[8 A]$ we refer to the maximal integer not exceeding $8 A$. For $j=1, \ldots,[8 A]+1, \xi \in F$, set $Q_{j}(\xi)=\{x \in$ $\left.Q(f),-A+\frac{(j-1) \epsilon}{8} \leq \xi^{*}(x)<-A+\frac{j \epsilon}{8}\right\}$. Let $\mathcal{B}:=\bigvee_{\xi \in F}\left\{Q_{1}(\xi), \ldots, Q_{[8 A]+1}(\xi)\right\}$ Then $\mathcal{B}$ is a partition of $Q(f)$.

For $\eta$ chosen above, define $\left\{\delta_{\ell}\right\}_{\ell \geq 1}, \delta_{\ell}<\eta$ by Lemma 3.1. Take an integer $\ell_{0}$ large enough so that $\tilde{\omega}\left(Q(f) \cap \tilde{\Lambda}_{\ell_{0}}\right)>1-\frac{\epsilon}{16}$ and $\omega\left(Q(f) \cap \tilde{\Lambda}_{\ell_{0}}\right)>1-\frac{\epsilon}{16}$. Also, take and fix for $\tilde{\Lambda}_{\ell_{0}}$ a finite cover $\alpha=\left\{U_{1}, \ldots, U_{r}\right\}$ by open balls in $M$ so that 
$\operatorname{diam}\left(U_{i}\right)<\delta_{\ell_{0}}$ and $\tilde{\omega}\left(U_{i} \cap \tilde{\Lambda}_{\ell_{0}}\right)>0, \quad i=1, \ldots, r$. We can assume without loss of generality that

$$
\omega\left(\bigcup_{j=1}^{r} U_{j}\right)=1
$$

Whenever an element $B$ in $\mathcal{B}$ is contained not inside a single set $U_{i}$ in $\alpha$ but instead in a union of open sets, say, $\bigcup_{j=1}^{\gamma} U_{j}$, we then divide $B$ into $\gamma$ subsets as follows:

$$
B_{1}=B \cap U_{1}, \quad B_{2}=B \cap\left(U_{2} \backslash U_{1}\right), \ldots, \quad B_{\gamma}=B \cap\left(U_{\gamma} \backslash \bigcup_{j=1}^{\gamma-1} U_{j}\right) .
$$

In this way we define a partition for $Q(f)$, denoted by $\left\{Q_{j}\right\}_{j=1}^{k}$. Observe that for each $Q_{j}$, there is $U_{j^{\prime}} \in \alpha$ such that $Q_{j} \subset U_{j^{\prime}}$ and thus $\operatorname{diam}\left(Q_{j}\right)<\delta_{\ell_{0}}, \quad j=1, \ldots, k$. Without loss of generality we may assume that $\omega\left(Q_{j}\right)>0$ for each $j=1, \ldots, k$ and thus may assume that $\omega\left(\bigcup_{j=1}^{k} Q_{j}\right)=1$.

Step II. A reduction of $\int_{Q(f)} \xi d \omega, \xi \in F$.

By using Poincaré's Recurrence Lemma, for $Q_{j}$ take a subset $N_{j} \subset Q_{j} \cap \tilde{\Lambda}_{\ell_{0}}$ such that

(1) $\omega\left(N_{j}\right)=\tilde{\omega}\left(N_{j}\right)=0$;

(2) for $x_{j} \in\left(Q_{j} \cap \tilde{\Lambda}_{\ell_{0}}\right) \backslash N_{j}$ there exists an integer $n_{j}$ so that $f^{n_{j}} x_{j} \in Q_{j} \cap \tilde{\Lambda}_{\ell_{0}}$;

$$
\frac{1}{n_{j}} \Sigma_{i=0}^{n_{j}-1} \xi\left(f^{i} x_{j}\right)=\xi^{*}\left(x_{j}\right)+\tau_{j}, \quad\left|\tau_{j}\right|<\frac{\epsilon}{8}, \quad j=1, \ldots, k .
$$

From Step I, $\left|\int_{Q(f)} \xi^{*} d \omega-\Sigma_{j=1}^{k} \int_{Q_{j}(f)} \xi^{*}\left(x_{j}\right) d \omega\right|<\frac{\epsilon}{8}$; thus we have

$$
\begin{aligned}
& \left|\int_{Q(f)} \xi d \omega-\Sigma_{j=1}^{k} \omega\left(Q_{j}\right) \frac{1}{n_{j}} \Sigma_{i=0}^{n_{j}-1} \xi\left(f^{i} x_{j}\right)\right| \\
& \quad=\left|\int_{Q(f)} \xi d \omega-\Sigma_{j=1}^{k} \omega\left(Q_{j}\right)\left(\xi^{*}\left(x_{j}\right)+\tau_{j}\right)\right| \\
& \quad<\frac{\epsilon}{8}+\frac{\epsilon}{8}=\frac{\epsilon}{4}, \quad \xi \in F .
\end{aligned}
$$

Step III. A further reduction of $\int_{Q(f)} \xi d \omega, \xi \in F$.

For $\eta>0$ as in (3.5) take $S>0$ such that $s \geq S$ implies $\frac{1}{s}<\frac{\eta}{k}$. Fix an integer $s \geq S$ and take $\bar{s}_{1}, \ldots, \bar{s}_{k}<s$ so that

$$
\frac{\bar{s}_{j}}{s} \leq \omega\left(Q_{j}\right) \leq \frac{\bar{s}_{j}+1}{s} .
$$

It follows by taking $s_{j}=\bar{s}_{j}$ or $\bar{s}_{j}+1$ that

$$
s=\Sigma_{j=1}^{k} s_{j}, \quad\left|\omega\left(Q_{j}\right)-\frac{s_{j}}{s}\right|<\frac{1}{s}<\frac{\eta}{k} .
$$


Thus from (3.7) and the choice of $\eta$ it follows that

$$
\begin{aligned}
& \left|\int_{Q(f)} \xi d \omega-\frac{1}{s} \sum_{j=1}^{k} s_{j} \frac{1}{n_{j}} \Sigma_{i=0}^{n_{j}-1} \xi\left(f^{i} x_{j}\right)\right| \\
& \quad \leq\left|\int_{Q(f)} \xi d \omega-\Sigma_{j=1}^{k} \omega\left(Q_{j}\right) \frac{1}{n_{j}} \sum_{i=0}^{n_{j}-1} \xi\left(f^{i} x_{j}\right)\right|+\frac{1}{s} k \\
& \quad=\frac{\epsilon}{4}+\eta<2 \frac{\epsilon}{4}=\frac{\epsilon}{2}, \quad \xi \in F .
\end{aligned}
$$

Step IV. Constructing a pseudo-orbit and a tracing periodic point.

Recall that $\tilde{\omega}$ is ergodic, and thus for each pair $(i, j), 1 \leq i, j \leq k$, there is an integer $X(i, j) \geq 1$ such that $f^{X(i, j)} Q_{i} \cap Q_{j} \cap \tilde{\Lambda}_{\ell_{0}} \neq \emptyset$. Take $y_{i} \in Q_{i} \cap \tilde{\Lambda}_{\ell_{0}}$ so that $f^{X(i, i+1)} y_{i} \in Q_{i+1} \cap \tilde{\Lambda}_{\ell_{0}}, 1 \leq i<k$ and $f^{X(k, 1)} y_{k} \in Q_{1} \cap \tilde{\Lambda}_{\ell_{0}}$. Recall that $x_{j}, f^{n_{j}}\left(x_{j}\right) \in Q_{j}$. Now we construct a periodic $\left\{\delta_{\ell}\right\}_{\ell \geq 1}$ pseudo-orbit by repeating the following sequence infinitely many times:

$$
\begin{gathered}
\underbrace{x_{1}, \ldots, f^{n_{1}-1} x_{1}, x_{1}, \ldots, f^{n_{1}-1} x_{1}, \ldots, x_{1}, \ldots, f^{n_{1}-1} x_{1}}_{s_{1} \text { times }}, y_{1}, \ldots, f^{X(1,2)-1} y_{1}, \\
\underbrace{x_{2}, \ldots, f^{n_{2}-1} x_{2}, x_{2}, \ldots, f^{n_{2}-1} x_{2}, \ldots, x_{2}, \ldots, f^{n_{2}-1} x_{2}}_{s_{2} \text { times }}, y_{2}, \ldots, f^{X(2,3)-1} y_{2}, \\
\ldots \ldots \quad \ldots \\
\underbrace{x_{k}, \ldots, f^{n_{k}-1} x_{k}, x_{k}, \ldots, f^{n_{k}-1} x_{k}, \ldots, x_{k}, \ldots, f^{n_{k}-1} x_{k}}_{s_{k} \text { times }}, y_{k}, \ldots, f^{X(k, 1)-1} y_{k} .
\end{gathered}
$$

By Corollary 3.5 there exists a hyperbolic periodic point $z \in \Lambda$ with period $p$, $\eta$-tracing this pseudo-orbit. Then

$$
p=\sum_{j=1}^{k} s_{j} n_{j}+X(1,2)+X(2,3)+\cdots+X(k-1, k)+X(k, 1) .
$$

Let

$$
\mu_{z}=\frac{1}{p} \Sigma_{i=0}^{p-1} \delta_{f^{i} z},
$$

where $\delta_{x}$ denotes the Dirac measure supported at the point $x$. Then

$$
\int \xi d \mu_{z}=\frac{1}{p} \Sigma_{i=0}^{p-1} \xi\left(f^{i} z\right), \quad \forall \xi \in F .
$$

Step V. Completing the proof.

We denote by $\left[x_{j}, f^{n_{j}-1} x_{j}\right]$ the orbit segment $x_{j}, \ldots, f^{n_{j}-1} x_{j}$ in (3.9) and let

$$
I=\bigcup_{j=1}^{k} \bigcup_{i=1}^{s_{j}}\left[x_{j}, f^{n_{j}} x_{j}\right] .
$$

Denote by $\Gamma$ the set

$$
\begin{gathered}
\left\{0,1, \ldots, n_{1}-1, n_{1}, \ldots, 2 n_{1}-1, \ldots,\left(s_{1}-1\right) n_{1}, \ldots, s_{1} n_{1}-1,\right. \\
s_{1} n_{1}+X(1,2), \ldots, s_{1} n_{1}+n_{2}+X(1,2)-1, \ldots, \\
s_{1} n_{1}+\left(s_{2}-1\right) n_{2}+X(1,2), \ldots, s_{1} n_{1}+s_{2} n_{2}+X(1,2)-1, \\
\ldots \ldots \ldots \ldots \ldots \ldots \ldots \ldots \ldots \ldots \ldots \ldots \ldots \ldots . . \\
\sum_{j=1}^{k-1} s_{j} n_{j}+\sum_{j=1}^{k-1} X(j, j+1), \ldots, \Sigma_{j=1}^{k-1} s_{j} n_{j}+\sum_{j=1}^{k-1} X(j, j+1)+n_{k}-1, \ldots, \\
\left.\sum_{j=1}^{k-1} s_{j} n_{j}+\sum_{j=1}^{k-1} X(j, j+1)+\left(s_{k}-1\right) n_{k}, \ldots, \sum_{j=1}^{k} s_{j} n_{j}+\sum_{j=1}^{k-1} X(j, j+1)-1\right\} .
\end{gathered}
$$


Then $\# I=\Sigma_{j=1}^{k} s_{j} n_{j}=\# \Gamma$. By (3.2), we have

$$
\left|\frac{1}{\# I} \Sigma_{x \in I} \xi(x)-\frac{1}{\# \Gamma} \Sigma_{i \in \Gamma} \xi\left(f^{i} z\right)\right| \leq \frac{\epsilon}{8} .
$$

Observe that $|\xi| \leq 1, \forall \xi \in F$; hence

$$
\left|\Sigma_{i=0}^{p-1} \xi\left(f^{i} z\right)-\Sigma_{i \in \Gamma} \xi\left(f^{i} z\right)\right| \leq k \max _{1 \leq i, j \leq r} X(i, j) .
$$

Thus we have

$$
\begin{aligned}
& \left|\frac{1}{p} \sum_{i=0}^{p-1} \xi\left(f^{i} z\right)-\frac{1}{\# I} \Sigma_{x \in I} \xi(x)\right| \\
& \quad \leq\left|\frac{1}{p} \Sigma_{i=0}^{p-1} \xi\left(f^{i} z\right)-\frac{1}{\# \Gamma} \Sigma_{i \in \Gamma} \xi\left(f^{i} z\right)\right|+\frac{\epsilon}{8} \\
& \quad=\left|\frac{1}{p \# \Gamma} \Sigma_{i=0}^{p-1}\left[\# \Gamma \xi\left(f^{i} z\right)-p \xi\left(f^{i} z\right)\right]+\frac{1}{\# \Gamma} \Sigma_{i \notin \Gamma} \xi\left(f^{i} z\right)\right|+\frac{\epsilon}{8} \\
& \quad \leq \frac{p(p-\# \Gamma)}{p \# \Gamma}+\frac{k \max _{1 \leq i, j \leq r} X(i, j)}{\# \Gamma}+\frac{\epsilon}{8} \leq \frac{2 k \max _{1 \leq i, j \leq r} X(i, j)}{\# \Gamma}+\frac{\epsilon}{8} .
\end{aligned}
$$

Hence

$$
\left|\frac{1}{p} \Sigma_{i=0}^{p-1} \xi\left(f^{i} z\right)-\frac{1}{\# I} \Sigma_{x \in I} \xi(x)\right|<\frac{\epsilon}{4}, \quad \xi \in F,
$$

by choosing $n_{j}$ large enough.

Without loss of generality, we assume $n_{j}$ are taken for $x_{j}$ by Lemma 3.7, $j=$ $1,2, \ldots, k$. It follows that

$$
\frac{s_{1}\left|n_{1}-n_{j}\right|+\cdots+s_{j-1}\left|n_{j-1}-n_{j}\right|+s_{k+1}\left|n_{j+1}-n_{j}\right|+\cdots+s_{k}\left|n_{k}-n_{j}\right|}{s \sum_{j=1}^{k} s_{j} n_{j}}
$$

is small. Observe that

$$
\begin{aligned}
& \left|\frac{1}{s} \sum_{j=1}^{k} s_{j} \frac{1}{n_{j}} \Sigma_{i=0}^{n_{j}-1} \xi\left(f^{i} x_{j}\right)-\frac{1}{\# I} \Sigma_{x \in I} \xi(x)\right| \\
& =\left|\sum_{j=1}^{k} \frac{s_{j}}{\left(s_{1}+\cdots+s_{k}\right)} \frac{1}{n_{j}} \Sigma_{i=0}^{n_{j}-1} \xi\left(f^{i} x_{j}\right)-\sum_{j=1}^{k} \frac{s_{j}}{s_{1} n_{1}+\cdots+s_{k} n_{k}} \Sigma_{i=0}^{n_{j}-1} \xi\left(f^{i} x_{j}\right)\right| \\
& =\mid \Sigma_{j=1}^{k} s_{j} \frac{s_{1}\left(n_{1}-n_{j}\right)+\cdots+s_{j-1}\left(n_{j-1}-n_{j}\right)+s_{j+1}\left(n_{j+1}-n_{j}\right)+\cdots+s_{k}\left(n_{k}-n_{j}\right)}{s \Sigma_{j=1}^{k} s_{j} n_{j}} \\
& \quad \cdot \frac{1}{n_{j}} \Sigma_{i=0}^{n_{j}-1} \xi\left(f^{i} x_{j}\right) \mid \\
& \leq\left|\sum_{j=1}^{k} s_{j} \frac{s_{1}\left(n_{1}-n_{j}\right)+\cdots+s_{j-1}\left(n_{j-1}-n_{j}\right)+s_{j+1}\left(n_{j+1}-n_{j}\right)+\cdots+s_{k}\left(n_{k}-n_{j}\right)}{s \Sigma_{j=1}^{k} s_{j} n_{j}}\right| .
\end{aligned}
$$

So we have

$$
\left|\frac{1}{s} \Sigma_{j=1}^{k} s_{j} \frac{1}{n_{j}} \Sigma_{i=0}^{n_{j}-1} \xi\left(f^{i} x_{j}\right)-\frac{1}{\# I} \Sigma_{x \in I} \xi(x)\right|<\frac{\epsilon}{4} .
$$

This together with (3.11) gives rise to the following inequality:

$$
\begin{aligned}
& \left|\frac{1}{p} \sum_{i=0}^{p-1} \xi\left(f^{i} z\right)-\frac{1}{s} \sum_{j=1}^{k} s_{k} \frac{1}{n_{j}} \Sigma_{i=0}^{n_{j}-1} \xi\left(f^{i} x_{j}\right)\right| \\
& \quad=\left|\frac{1}{p} \Sigma_{i=0}^{p-1} \xi\left(f^{i} z\right)-\frac{1}{\# I} \Sigma_{x \in I} \xi(x)\right|+\frac{\epsilon}{4} \\
& \quad<\frac{\epsilon}{2}, \quad \xi \in F .
\end{aligned}
$$


Combining (3.8) with (3.13) we obtain that

$$
\begin{aligned}
\left|\int_{Q(f)} \xi d \omega-\int \xi d \mu_{z}\right| & \leq\left|\frac{1}{s} \sum_{j=1}^{k} s_{j} \frac{1}{n_{j}} \sum_{i=0}^{n_{j}-1} \xi\left(f^{i} x_{j}\right)-\frac{1}{p} \sum_{i=0}^{p-1} \xi\left(f^{i} z\right)\right|+\frac{\epsilon}{2} \\
& \leq \frac{\epsilon}{2}+\frac{\epsilon}{2}=\epsilon, \quad \forall \xi \in F .
\end{aligned}
$$

This completes the proof.

\section{Approximation properties on mean angle, INDEPENDENCE NUMBER AND OSELEDEC SPLITTINGS}

In this section we prove Theorem 1.12. We first introduce a theorem due to Sun and Vargas [17] which is one of the keys in the proof of Theorem 1.12.

Recall that $\mathcal{U}_{\ell}^{\#}$ is a bundle of unit $\ell$-frames, $\pi: \mathcal{U}_{\ell}^{\#} \rightarrow M$ is the canonical bundle projection and $\mathcal{M}_{\text {erg }}\left(\mathcal{U}_{\ell}^{\#}, D^{\#} f\right)$ is the set of all $D^{\#} f$-invariant and ergodic measures on $\mathcal{U}_{\ell}^{\#}, 1 \leq \ell \leq d=\operatorname{dim} M$.

Theorem 4.1 (17]). Let $f: M \rightarrow M$ be a $C^{1}$ diffeomorphism preserving an ergodic measure $\nu$ with simple spectrum. For a given integer $\ell, 1 \leq \ell \leq d=\operatorname{dim} M$, denote $\mathcal{B}:=\left\{\mu \in \mathcal{M}_{\text {erg }}\left(\mathcal{U}_{\ell}^{\#}, D^{\#} f\right) \mid \pi_{*}(\mu)=\nu\right\}$. Then

$$
A_{d}^{\ell} \leq \operatorname{Card}(\mathcal{B}) \leq 2^{d} A_{d}^{\ell},
$$

where $A_{d}^{\ell}=d(d-1) \cdots(d-\ell+1)$.

Recall that $L_{d}^{\#}=\mathcal{U}_{d}^{\#} \cup \mathcal{V}_{d}^{\#}$. Now we discuss the relation between the Oseledec splitting of a measure $\nu \in \mathcal{M}_{\text {erg }}(M, f)$ and a generic frame of a measure $\mu \in$ $\mathcal{M}_{\text {erg }}\left(L_{d}^{\#}, D^{\#} f\right)$ covering $\nu$, i.e., $\pi_{*}(\mu)=\nu$. Suppose $\nu$ has a simple spectrum and its Oseledec splitting is

$$
T M=E_{1} \oplus E_{2} \oplus \cdots \oplus E_{d}, d=\operatorname{dim} M .
$$

We claim that for any point $x \in O(\nu)$, the Oseledec basin of $\nu$, and any frame $\alpha=\left(u_{1}(x), \ldots, u_{d}(x)\right) \in Q_{\mu}\left(L_{d}^{\#}, D^{\#} f\right)$, it holds that $u_{i}(x) \in E_{j(i)}(x)$ for some $j(i), \forall i=1, \ldots, d$. Indeed, we define $\phi_{i}: L_{d}^{\#} \rightarrow \mathbb{R}$ by

$$
\phi_{i}(\beta)=\log \left\|\pi_{i} D f(\beta)\right\|, \quad \beta \in L_{d}^{\#},
$$

where $\pi_{i}$ denotes the projection from a frame to its $i$-th vector, $i=1,2, \ldots, d$. Then all these functions $\phi_{i}$ are clearly bounded and continuous. By the definition of $Q_{\mu}\left(L_{d}^{\#}, D^{\#} f\right)$ and the Birkhoff Ergodic Theorem, we have

$$
\lim _{n \rightarrow \pm \infty} \frac{1}{n} \log \left\|D f^{n}\left(u_{i}\right)\right\|=\lim _{n \rightarrow \pm \infty} \frac{1}{n} \sum_{j=1}^{n} \phi_{i}\left(D^{\#} f^{j} \alpha\right)=\int \phi_{i} d \mu,
$$

which implies that $u_{i} \in E_{j(i)}$ for some $j(i)$, and thus the claim follows.

In light of Theorem 4.1 and this claim, it seems reasonable to classify all the ergodic measures on $\mathcal{U}_{d}^{\#}$ which cover an ergodic measure with a simple spectrum. Since the measures supported on $\mathcal{V}_{d}^{\#}$ make no contribution to mean angles or independence numbers (for details, see Step II in the proof of Theorem 1.12(1)), we will take into account the measures on $\mathcal{U}_{d}^{\#}$ only. In order to make a precise classification in the proof of Theorem 1.12, we need a new notion as follows. 
Definition 4.2. Let $\nu$ be an ergodic measure on $M$ with simple spectrum:

$$
\lambda_{1}<\lambda_{2}<\cdots<\lambda_{d}, \quad d=\operatorname{dim} M .
$$

An ergodic measure $\mu \in \mathcal{M}_{\text {erg }}\left(\mathcal{U}_{d}^{\#}, D^{\#} f\right)$ covering $\nu$ is said to be $(\sigma(1), \sigma(2), \ldots$, $\sigma(d))$-type if

$$
\int_{\mathcal{U}_{d}^{\#}} \log \left\|\pi_{i} D f\right\| d \mu=\lambda_{\sigma(i)}, \quad i=1,2, \ldots, d,
$$

where $\sigma:\{1,2, \ldots, d\} \rightarrow\{1,2, \ldots, d\}$ is a permutation.

Remark. If $\mu$ is $(\sigma(1), \sigma(2), \ldots, \sigma(d))$-type, by the claim before Definition 4.2, one can deduce that any frame $\alpha=\left(u_{1}, u_{2}, \ldots, u_{d}\right)$ in the basin $Q_{\mu}\left(\mathcal{U}_{d}^{\#}, D^{\#} f\right)$ must satisfy that $u_{i} \in E_{\sigma(i)}$ for any $i=1,2, \ldots, d$, where

$$
T M=E_{1} \oplus E_{2} \oplus \cdots \oplus E_{d}
$$

is the Oseledec splitting of $\nu$. We also say $\alpha$ to be of $(\sigma(1), \sigma(2), \ldots, \sigma(d))$-type in this case.

Proof of Theorem 1.12, Let $\varepsilon=\frac{1}{n}, n \geq 1$. Applying Theorem 1.9 and Theorem 1.8 to the ergodic measure $\tilde{\omega}$, we can find a hyperbolic periodic point $z_{n}$ with period $p_{n}$ which satisfies the following properties:

(a) the Lyapunov exponents of $z_{n}$ are $\varepsilon$-close to those of the measure $\tilde{\omega}$ in the sense that after arranging all the Lyapunov exponents of $z_{n}$ and those of $\tilde{\omega}$ in increasing order, respectively, the $i$-th exponent of $z_{n}$ and the $i$-th one of $\tilde{\omega}$ are $\varepsilon$-close, $i=1,2, \ldots, d$;

(b) the atomic measure $\omega_{n}=\frac{1}{p_{n}} \sum_{i=1}^{p_{n}-1} \delta_{f^{i} z_{n}}$ is $\varepsilon$-close to the measure $\tilde{\omega}$ in the weak*-topology.

Without loss of generality we assume that $\omega_{n}$ has a simple spectrum as $\tilde{\omega}$ has for all $n \geq 1$.

Proof of Theorem 1.12(1). We need to verify that there exists a convergence subsequence $\left\{z_{n_{k}}\right\}_{k \geq 1}$ such that

$$
\lim _{k \rightarrow \infty} \frac{1}{p_{n_{k}}} \sum_{m=0}^{p_{n_{k}}-1} \angle\left(E_{i}\left(f^{m}\left(z_{n_{k}}\right)\right), E_{t}\left(f^{m}\left(z_{n_{k}}\right)\right)\right)=m \angle_{\tilde{\omega}}\left(E_{i}, E_{t}\right), \quad 1 \leq i \neq t \leq d,
$$

where $E_{1}\left(f^{m}\left(z_{n_{k}}\right)\right) \oplus \cdots \oplus E_{d}\left(f^{m}\left(z_{n_{k}}\right)\right)$ is the Oseledec splitting at $f^{m}\left(z_{n_{k}}\right)$.

We divide the proof into three steps.

Step I. Finding an appropriate invariant measure $\mu$ on $L_{d}^{\#}$ to cover the measure $\tilde{\omega}$.

Choose an ergodic measure $\mu_{n} \in \mathcal{M}_{\text {erg }}\left(\mathcal{U}_{d}^{\#}, D^{\#} f\right)$ covering $\omega_{n}$ according to Theorem 2.7. Since $\mathcal{U}_{d}^{\#} \subseteq L_{d}^{\#}$ and $L_{d}^{\#}$ is compact, we can assume that $\mu_{n}$ converges to an invariant measure $\mu \in \mathcal{M}_{i n v}\left(L_{d}^{\#}, D^{\#} f\right)$ by taking subsequence if necessary. Then $\mu$ is a covering measure of $\tilde{\omega}$. That is, $\pi_{*} \mu=\tilde{\omega}$, since given any continuous 
function $\xi: M \rightarrow \mathbb{R}$, it follows that

$$
\begin{aligned}
\int \xi d \tilde{\omega} & = & \lim _{n \rightarrow+\infty} \int \xi d \omega_{n} \\
& = & \lim _{n \rightarrow+\infty} \int \xi d \pi_{*} \mu_{n} \\
& = & \lim _{n \rightarrow+\infty} \int \xi \circ \pi d \mu_{n} \\
& = & \int \xi \circ \pi d \mu \\
& = & \int \xi d \pi_{*} \mu .
\end{aligned}
$$

Therefore, we get the following commutative graph:

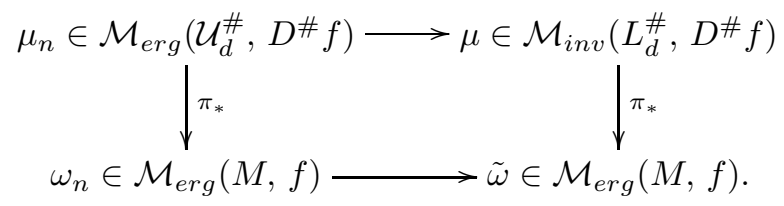

By the Birkhoff Ergodic Theorem,

$$
\int \phi d \mu=\lim _{n \rightarrow+\infty} \int \phi d \mu_{n}=\lim _{n \rightarrow+\infty} \lim _{j \rightarrow+\infty} \frac{1}{j} \sum_{i=0}^{j-1} \phi\left(D^{\#} f^{i} \alpha_{z_{n}}\right),
$$

$\forall \alpha_{z_{n}} \in Q_{\mu_{n}}\left(\mathcal{U}_{d}^{\#}, D^{\#} f\right), \forall \phi \in C^{b}\left(\mathcal{U}_{d}^{\#}, \mathbb{R}\right)$.

Step II. Analyzing the number and type of the ergodic measures decomposed from $\mu$.

Let

$$
\mathcal{A}:=\left\{\nu \in \mathcal{M}_{\text {erg }}\left(\mathcal{U}_{d}^{\#}, D^{\#} f\right) \mid \pi_{*}(\nu)=\tilde{\omega}\right\} .
$$

Claim. The ergodic measures $\nu$ decomposed from $\mu$ are all supported on the bundle $\mathcal{U}_{d}^{\#}$, i.e., $\nu \in \mathcal{A}$.

Proof. Denote $H(\cdot): L^{\#}(M) \rightarrow \mathcal{F}^{\#}(M)$ to be the Gram-Schmidt orthogonalization process and normalization where $L^{\#}(M)=L_{1}^{\#}(M) \cup \cdots \cup L_{d}^{\#}(M)$ and $\mathcal{F}^{\#}(M)=$ $\mathcal{F}_{1}^{\#}(M) \cup \cdots \cup \mathcal{F}_{d}^{\#}(M)$. Each bundle $\mathcal{F}_{i}^{\#}(M)$, a subset of $L_{i}^{\#}(M)$, consists of all the orthogonal frames with $i$ vectors, $1 \leq i \leq d$.

Denote $\omega=\left(\omega_{0}, \omega_{1}, \ldots, \omega_{n}, \ldots\right)$ where $\omega_{0}=\tilde{\omega}$. For $1 \leq k \leq d=\operatorname{dim} M$, let

$$
\begin{aligned}
& \mathcal{F}_{k}^{\#}(\omega) \\
& \quad:=\left\{\alpha=\left(u_{1}, \ldots, u_{k}\right) \in \mathcal{F}_{k}^{\#}(M)|| u_{i} \mid=1, \exists \beta=\left(v_{1}, \ldots, v_{k}\right) \in \mathcal{U}_{k}^{\#}(M), \exists n \geq 0,\right.
\end{aligned}
$$

s.t. $\beta$ is a quasi-regular point of some covering measure of $\omega_{n}$ and $\left.\alpha=H(\beta)\right\}$.

Note that each $\omega_{n}$ has a simple spectrum and thus its style number is $d$, and note $k \leq d$. Theorem 2.7 ensures the existence of ergodic measures on $\mathcal{U}_{k}^{\#}(M)$ covering $\omega_{n}, n \geq 0$. Hence the bundle $\mathcal{F}_{k}^{\#}(\omega)$ is well defined. Let

$L_{k}^{\#}(\omega):=\left\{\alpha=\left(u_{1}, \ldots, u_{k}\right) \in L_{k}^{\#}(M)|| u_{i} \mid=1, \exists n \geq 0\right.$, s.t.

$u_{i}$ is contained in some Oseledec invariant bundle of $\left.\omega_{n}, \forall i=1,2, \ldots, k\right\}$.

The vectors of any frame in $L_{k}^{\#}(\omega)$ are not necessarily taken from different Oseledec bundles. Denote

$$
L^{\#}(\omega)=L_{1}^{\#}(\omega) \cup \cdots \cup L_{d}^{\#}(\omega) \quad \text { and } \quad \mathcal{F}^{\#}(\omega)=\mathcal{F}_{1}^{\#}(\omega) \cup \cdots \cup \mathcal{F}_{d}^{\#}(\omega) .
$$


They are two bundles supported on $\bigcup_{i=0}^{\infty} Q_{\omega_{i}}(M, f)$ where $Q_{\omega_{i}}(M, f)$ is the basin of $\omega_{i}, i \geq 0$. Also, let

$$
\chi^{\#}=\left.H \circ D f\right|_{\mathcal{F}^{\#}(\omega)}: \mathcal{F}^{\#}(\omega) \rightarrow \mathcal{F}^{\#}(\omega) .
$$

Then we get a commutative graph:

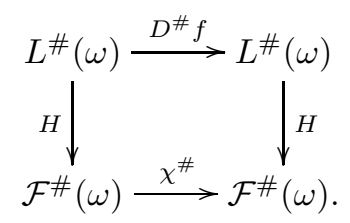

It is clear that $H: L^{\#}(\omega) \rightarrow \mathcal{F}^{\#}(\omega)$ is a continuous surjection and the measures $\mu_{n}$ and $\mu$ are all supported on the bundle $L^{\#}(\omega)$. Let

$$
\tilde{\mu}_{n}:=H_{*} \mu_{n} \quad \text { and } \quad \tilde{\mu}:=H_{*} \mu .
$$

Then they are all supported on $\mathcal{F}^{\#}(\omega)$ and $\tilde{\mu}_{n} \rightarrow \tilde{\mu}$ as $n \rightarrow \infty$. Moreover, since $\mu_{n}$ are supported on $\mathcal{U}_{d}^{\#}$, it must hold that $\tilde{\mu}_{n}$ are supported on $\mathcal{F}_{d}^{\#}(\omega)$. Note that $\tilde{\mu}_{n}$ covers $\omega_{n}$ and $\tilde{\mu}$ covers $\tilde{\omega}$, respectively, and $\omega_{n} \rightarrow \tilde{\omega}$ as $n \rightarrow+\infty$. We can deduce that $\tilde{\mu}$ is supported on $\left.\mathcal{F}_{d}^{\#}\right|_{Q_{\tilde{\omega}}(M, f)}$.

According to the Ergodic Decomposition Theorem and the result in [17] (the same result as Theorem 4.1 for $\mathcal{F}^{\#}(M)$ ), there exist finitely many ergodic measures $\tilde{m}_{1}, \ldots, \tilde{m}_{\ell} \in \mathcal{M}_{\text {erg }}\left(\left.\mathcal{F}_{d}^{\#}\right|_{Q_{\tilde{\omega}}(M, f)}, \chi^{\#}\right)$ and positive real numbers $a_{1}, \ldots, a_{\ell}$ with $\sum_{i=1}^{\ell} a_{i}=1\left(1 \leq \ell \leq 2^{d} d !\right)$ such that

$$
\tilde{\mu}=a_{1} \tilde{m}_{1}+\cdots+a_{\ell} \tilde{m}_{\ell} .
$$

Since $H^{-1}\left(\left.\mathcal{F}_{d}^{\#}\right|_{Q_{\tilde{\omega}}(M, f)}\right) \subseteq \mathcal{U}_{d}^{\#}(M)$, all the measures covering $\tilde{\mu}$ must be supported on $\mathcal{U}_{d}^{\#}(M)$. Especially, we have that $\mu \in \mathcal{M}_{\text {erg }}\left(\mathcal{U}_{d}^{\#}(M)\right)$. Hence we proved the claim.

Combining the claim with Theorem 4.1, the number of ergodic measures decomposed from $\mu$ is not more than $2^{d} d$ ! and they are all supported on $\mathcal{U}_{d}^{\#}$. Since adding ergodic measures weighing vacancy to the sum of all ergodic measures decomposed from $\mu$ will not have any effect, we assume that the set of ergodic measures decomposed from $\mu$ coincides with $\mathcal{A}$ and $\operatorname{Card} \mathcal{A}=2^{d} d$ !. Now we arrange all these $2^{d} d$ ! measures, denoted by $\nu_{j}, j=1,2, \ldots, 2^{d} d$ !, according to their types (see Definition 4.2).

We first divide all these $2^{d} d$ ! measures into $d$ sets, each having $2^{d}(d-1)$ ! members and the $\ell$-th set consisting of all the measures of $(\ell, *, *, \cdots *)$-type, $\ell=1,2, \ldots, d$. Specifically, the $\ell$-th set is

$\left\{\nu_{j} \in \mathcal{A} \mid \nu_{j}\right.$ is of $(\ell, *, *, \cdots *)$ - type $\left., j=(\ell-1) \cdot 2^{d}(d-1) !+1, \ldots, \ell \cdot 2^{d}(d-1) !\right\}$,

where $(\ell-1) \cdot 2^{d}(d-1)$ ! and $\ell \cdot 2^{d}(d-1)$ ! denote the sum of the cardinalities of the first $\ell-1$ sets and the sum of the cardinalities of the first $\ell$ sets, respectively.

Further, we divide each set into $d-1$ subsets with cardinalities all being $2^{d}(d-2)$ ! such that the $i$-th subset of the $\ell$-th set contains all the measures of $(\ell, i, *, *$, $\cdots, *$ )-type, $i=1,2, \ldots, d$, and $i \neq \ell$ (a measure should be supported on $\mathcal{V}_{d}^{\#}=$ 
$L_{d}^{\#}-\mathcal{U}_{d}^{\#}$ if it is $(\ell, \ell, *, *, \cdots *)$-type). More precisely, the $i$-th subset of the $\ell-$ th set is

$$
\left\{\nu_{j} \in \mathcal{A} \mid \nu_{j} \text { is of }(\ell, i, *, *, \cdots *) \text { - type },\right.
$$

$\left.j=(\ell-1) \cdot 2^{d}(d-1) !+(i-1) 2^{d}(d-2) !+1, \ldots,(\ell-1) \cdot 2^{d}(d-1) !+i \cdot 2^{d}(d-2) !\right\}$.

Here the number $(i-1) 2^{d}(d-2)$ ! denotes the sum of the cardinalities of the first $i-1$ subsets in the $\ell-$ th set, and hence the number $(\ell-1) \cdot 2^{d}(d-1) !+(i-1) 2^{d}(d-2)$ ! denotes the sum of the cardinalities of the first $\ell-1$ sets and the cardinalities of these $i-1$ subsets. Analogically, one explains the number $(\ell-1) \cdot 2^{d}(d-1) !+i \cdot 2^{d}(d-2)$ !.

By repeating this procedure, one can divide $\mathcal{A}$ into $d$ ! disjoint classes, each containing $2^{d}$ measures. The question as to which class a measure in $\mathcal{A}$ belongs to is uniquely determined by its type. Specially, the first $2^{d}$ measures, $\nu_{j}, j=$ $1,2, \ldots, 2^{d}$, are of $(1,2,3, \ldots, d)$-type (here ' $\ldots$ ' represents the sequence of integers from 4 to $d-1$ in increasing order).

Using the Ergodic Decomposition Theorem, there exists a function $\iota: \mathcal{A} \rightarrow[0,1]$ such that $\sum_{\nu \in \mathcal{A}} \iota(\nu)=1$ and

$$
\int \phi d \mu=\sum_{j=1}^{2^{d} d !}\left(\int_{Q_{\nu_{j}}\left(\mathcal{U}_{d}^{\#}, D^{\#} f\right)} \phi d \nu_{j}\right) \iota\left(\nu_{j}\right), \quad \forall \phi \in C^{b}\left(\mathcal{U}_{d}^{\#}, \mathbb{R}\right),
$$

where $Q_{\nu_{j}}\left(\mathcal{U}_{d}^{\#}, D^{\#} f\right)$ denotes the basin of $\nu_{j}$ (see Section 1 for the definition). Let

$$
a_{j}=\iota\left(\nu_{j}\right), \quad 1 \leq j \leq 2^{d} d ! .
$$

Applying the Birkhoff Ergodic Theorem to $\nu_{j}$ in (4.2), it holds for any $\alpha_{j} \in$ $Q_{\nu_{j}}\left(\mathcal{U}_{d}^{\#}, D^{\#} f\right)$ that

$$
\int \phi d \mu=\sum_{j=1}^{2^{d} d !} a_{j}\left(\lim _{n \rightarrow+\infty} \frac{1}{n} \sum_{i=0}^{n-1} \phi\left(D^{\#} f^{i}\left(\alpha_{j}\right)\right)\right),
$$

for all $\phi \in C^{b}\left(\mathcal{U}_{d}^{\#}, \mathbb{R}\right)$. Combining (4.1) with (4.2), we have

$$
\lim _{n \rightarrow+\infty} \lim _{j \rightarrow+\infty} \frac{1}{j} \sum_{i=0}^{j-1} \phi\left(D^{\#} f^{i} \alpha_{z_{n}}\right)=\sum_{j=1}^{2^{d} d !} a_{j}\left(\lim _{n \rightarrow+\infty} \frac{1}{n} \sum_{i=0}^{n-1} \phi\left(D^{\#} f^{i}\left(\alpha_{j}\right)\right),\right.
$$

for all $\phi \in C^{b}\left(\mathcal{U}_{d}^{\#}, \mathbb{R}\right), \alpha_{z_{n}} \in Q_{\mu_{n}}\left(\mathcal{U}_{d}^{\#}, D^{\#} f\right), \alpha_{j} \in Q_{\nu_{j}}\left(\mathcal{U}_{d}^{\#}, D^{\#} f\right)$.

We need the following proposition, saying that there are at most $2^{d}$ ergodic measures summing up in the right hand of (4.2).

Proposition 4.3. Under the conditions of Theorem 1.12, the number of the ergodic measures decomposed from $\mu$ is not more than $2^{d}$ and these ergodic measures are of the same type.

Proof. Take $\phi_{t}$ for $\phi$ in (4.4), where $\phi_{t}, t=1,2, \ldots, d$, are defined before Definition 4.2, Then

$$
\lim _{n \rightarrow+\infty} \lim _{j \rightarrow+\infty} \frac{1}{j} \sum_{i=0}^{j-1} \phi_{t}\left(D^{\#} f^{i} \alpha_{z_{n}}\right)=\sum_{j=1}^{2^{d} d !} a_{j}\left(\lim _{n \rightarrow+\infty} \frac{1}{n} \sum_{i=0}^{n-1} \phi_{t}\left(D^{\#} f^{i}\left(\alpha_{j}\right)\right)\right) .
$$

Denote by $\left\{\lambda_{1}\left(z_{n}\right)<\lambda_{2}\left(z_{n}\right)<\cdots<\lambda_{d}\left(z_{n}\right)\right\}$ and $\left\{\lambda_{1}<\lambda_{2}<\cdots<\lambda_{d}\right\}$ the Lyapunov spectrum of $\omega_{n}$ and that of $\tilde{\omega}$, respectively. We have $\lim _{n \rightarrow+\infty} \lambda_{t}\left(z_{n}\right)=\lambda_{t}$, 
$t=1, \ldots, d$, by Theorem 1.9. An analogous discussion as in the above claim before Definition 4.2 shows that the limit $\lim _{j \rightarrow+\infty} \frac{1}{j} \sum_{i=0}^{j-1} \phi_{t}\left(D^{\#} f^{i} \alpha_{z_{n}}\right)$ coincides with some Lyapunov exponent of $\omega_{n}$, assumed to be $\lambda_{t}\left(z_{n}\right)$, the $t$-th one, $t=1,2, \ldots, d$. Combining with (4.5), we obtain

$$
\lambda_{t}=\sum_{j=1}^{2^{d} d !} a_{j}\left(\lim _{n \rightarrow+\infty} \frac{1}{n} \sum_{i=0}^{n-1} \phi_{t}\left(D^{\#} f^{i}\left(\alpha_{j}\right)\right)\right) .
$$

Since $\tilde{\omega}$ has a simple spectrum and $\nu_{j}$ is supported on $\mathcal{U}_{d}^{\#}$, it holds that $\int \phi_{t_{1}} d \nu_{j}$ and $\int \phi_{t_{2}} d \nu_{j}$ are different for any $1 \leq t_{1} \neq t_{2} \leq d$. A similar argument for $\nu_{j}$ as in the above claim shows that $\lim _{n \rightarrow+\infty} \frac{1}{n} \sum_{i=0}^{n-1} \phi_{t}\left(D^{\#} f^{i}\left(\alpha_{j}\right)\right)$ coincides with the Lyapunov exponent of the $t$-th vector in the frame $\alpha_{j} \in Q_{\nu_{j}}\left(\mathcal{U}_{d}^{\#}, D^{\#} f\right)$. In the following we will give a detailed computation on (4.6) when $t$ varies from 1 to $d$ and then complete the proposition inductively.

When $t=1$, we have

$\lambda_{1}=\lambda_{1} \sum_{j=1}^{2^{d}(d-1) !} a_{j}+\lambda_{2} \sum_{j=2^{d}(d-1) !+1}^{2 \cdot 2^{d}(d-1) !} a_{j}+\lambda_{3} \sum_{j=2 \cdot 2^{d}(d-1) !+1}^{3 \cdot 2^{d}(d-1) !} a_{j}+\cdots+\lambda_{d} \sum_{j=(d-1) \cdot 2^{d}(d-1) !+1}^{2^{d} d !} a_{j}$,

i.e.,

$\lambda_{1}\left(1-\sum_{j=1}^{2^{d}(d-1) !} a_{j}\right)=\lambda_{2} \sum_{j=2^{d}(d-1) !+1}^{2 \cdot 2^{d}(d-1) !} a_{j}+\lambda_{3} \sum_{j=2 \cdot 2^{d}(d-1) !+1}^{3 \cdot 2^{d}(d-1) !} a_{j}+\cdots+\lambda_{d} \sum_{j=(d-1) \cdot 2^{d}(d-1) !+1}^{2^{d} d !} a_{j}$.

Since $\sum_{j=1}^{2^{d} d !} a_{j}=1$, it follows that

$$
\begin{aligned}
& \lambda_{1} \sum_{j=2^{d}(d-1) !+1}^{2 \cdot 2^{d}(d-1) !} a_{j}+\lambda_{1} \sum_{j=2 \cdot 2^{d}(d-1) !+1}^{3 \cdot 2^{d}(d-1) !} a_{j}+\cdots+\lambda_{1} \sum_{j=(d-1) \cdot 2^{d}(d-1) !+1}^{2^{d} d !} a_{j} \\
& \quad=\lambda_{2} \sum_{j=2^{d}(d-1) !+1}^{2 \cdot 2^{d}(d-1) !} a_{j}+\lambda_{3} \sum_{j=2 \cdot 2^{d}(d-1) !+1}^{3 \cdot 2^{d}(d-1) !} a_{j}+\cdots+\lambda_{d} \sum_{j=(d-1) \cdot 2^{d}(d-1) !+1}^{2^{d} d !} a_{j} .
\end{aligned}
$$

Thus we have

$$
\left(\lambda_{1}-\lambda_{2}\right) \sum_{j=2^{d}(d-1) !+1}^{2 \cdot 2^{d}(d-1) !} a_{j}+\left(\lambda_{1}-\lambda_{3}\right) \sum_{j=2 \cdot 2^{d}(d-1) !+1}^{3 \cdot 2^{d}(d-1) !} a_{j}+\cdots+\left(\lambda_{1}-\lambda_{d}\right) \sum_{j=(d-1) \cdot 2^{d}(d-1) !+1}^{2^{d} d !} a_{j}=0 .
$$

Since $\lambda_{1}<\lambda_{2}<\cdots<\lambda_{d}$, and $0 \leq a_{j} \leq 1,1 \leq j \leq 2^{d} d$ !, (4.7) implies that

$$
\sum_{j=2^{d}(d-1) !+1}^{2 \cdot 2^{d}(d-1) !} a_{j}=\sum_{j=2 \cdot 2^{d}(d-1) !+1}^{3 \cdot 2^{d}(d-1) !} a_{j}=\cdots=\sum_{j=(d-1) \cdot 2^{d}(d-1) !+1}^{2^{d} d !} a_{j}=0 .
$$

So

and thus

$$
a_{j}=0, \quad \forall j \geq 2^{d}(d-1) !+1
$$

$$
\sum_{j=1}^{2^{d}(d-1) !} a_{j}=1
$$


When $t=2$, we have by (4.8) that

$$
\lambda_{2}=\lambda_{2} \sum_{j=1}^{2^{d}(d-2) !} a_{j}+\lambda_{3} \sum_{j=2^{d}(d-2) !+1}^{2 \cdot 2^{d}(d-2) !} a_{j}+\lambda_{4} \sum_{j=2 \cdot 2^{d}(d-2) !+1}^{3 \cdot 2^{d}(d-2) !} a_{j}+\cdots+\lambda_{d} \sum_{j=(d-2) \cdot 2^{d}(d-2) !}^{2^{d}(d-1) !} a_{j} .
$$

From (4.8), a similar argument as in (4.7) shows that

$$
\left(\lambda_{2}-\lambda_{3}\right) \sum_{j=2^{d}(d-2) !+1}^{2 \cdot 2^{d}(d-2) !} a_{j}+\left(\lambda_{2}-\lambda_{4}\right) \sum_{j=2 \cdot 2^{d}(d-2) !+1}^{3 \cdot 2^{d}(d-2) !} a_{j}+\cdots+\left(\lambda_{2}-\lambda_{d}\right) \sum_{j=(d-2) \cdot 2^{d}(d-2) !}^{2^{d}(d-1) !} a_{j}=0 .
$$

Since $\lambda_{2}<\cdots<\lambda_{d}$, we have

$$
\sum_{j=2^{d}(d-2) !+1}^{2 \cdot 2^{d}(d-2) !} a_{j}=\sum_{j=2 \cdot 2^{d}(d-2) !+1}^{3 \cdot 2^{d}(d-2) !} a_{j}=\cdots=\sum_{j=(d-2) \cdot 2^{d}(d-2) !}^{2^{d}(d-1) !} a_{j}=0 .
$$

Thus the following equations are true:

$$
\sum_{j=1}^{2^{d}(d-2) !} a_{j}=1 \text { and } a_{j}=0, \quad j \geq 2^{d}(d-2) !+1 .
$$

Proceeding analogously to consider the case for $t=3,4, \ldots, d$, we can finally prove that

$$
\sum_{j=1}^{2^{d}} a_{j}=1 \text { and } a_{j}=0, \quad j \geq 2^{d}+1 .
$$

Hence, the proposition is proved.

Proposition 4.3 tells us that the sum in (4.2) is actually taken for $2^{d}$ ergodic measures all having the same type. Hence we finish the proof of Step II, and in the next step we focus on these $2^{d}$ ergodic measures.

Remark. In the proof of Proposition 4.3 we assumed that the limit $\lim _{j \rightarrow+\infty} \frac{1}{j} \sum_{i=0}^{j-1} \phi_{t}\left(D^{\#} f^{i} \alpha_{z_{n}}\right)$ coincides with $\lambda_{t}\left(z_{n}\right)$, the $t$-th Lyapunov exponent of $\omega_{n}$, where $\phi_{t}$ and $\lambda_{t}\left(z_{n}\right)$ share the same index $t, t=1,2, . ., d$. The other cases without this assumption can be proved similarly. For instance, assume $d=\operatorname{dim} M=3$ and

$$
\lim _{n \rightarrow+\infty} \lim _{j \rightarrow+\infty} \frac{1}{j} \sum_{i=0}^{j-1} \phi_{t}\left(D^{\#} f^{i} \alpha_{z_{n}}\right)=\lambda_{2 t} \bmod (3) \quad\left(\text { here let } \lambda_{0}=\lambda_{3}\right),
$$

$t=1,2,3$, where we recall $\lambda_{1}<\lambda_{2}<\lambda_{3}$. We first discuss the case when $t=2$ (i.e., the above limit is $\lambda_{1}$ ), then $t=1$ (the above limit is $\lambda_{2}$ ) and finally the case when $t=3$ (the above limit is $\lambda_{3}$ ), one after another. Then we can also prove that there are not more than $2^{3}$ ergodic measures decomposed from $\mu$ and they are all $(2,1,3)$-type.

Step III. Completing the proof of (1).

Define $\phi_{0}: \mathcal{U}_{2}^{\#} \rightarrow \mathbb{R},\left(u_{1}, u_{2}\right) \mapsto \angle\left(u_{1}, u_{2}\right)$. Clearly, $\phi_{0}$ is a bounded continuous function on $\mathcal{U}_{2}^{\#}$. For $i, j \in\{1,2, \ldots, d\}$, we define $\pi_{i j}: \mathcal{U}_{d}^{\#} \rightarrow \mathcal{U}_{2}^{\#}$, $\left(u_{1}, u_{2}, \ldots, u_{d}\right) \mapsto\left(u_{i}, u_{j}\right)$, and $\phi_{i j}: \mathcal{U}_{d}^{\#} \rightarrow \mathbb{R}, \phi_{i j}(\alpha)=\phi_{0}\left(\pi_{i j}(\alpha)\right)$. 
Let $\phi=\phi_{i t}, 1 \leq i \neq t \leq d$ in (4.4); then by $(4.10)$

$$
\lim _{n \rightarrow+\infty} \frac{1}{p_{n}} \sum_{s=0}^{p_{n}-1} \phi_{i t}\left(D^{\#} f^{s} \alpha_{z_{n}}\right)=\sum_{j=1}^{2^{d}} a_{j}\left(\lim _{n \rightarrow+\infty} \frac{1}{n} \sum_{s=0}^{n-1} \phi_{i t}\left(D^{\#} f^{s}\left(\alpha_{j}\right)\right)\right) .
$$

Since all the $2^{d}$ measures $\nu_{j}$ are of the same type by Proposition 4.3 and thus all the $2^{d}$ frames $\alpha_{j}$ in (4.11) are of the same type by the Remark following Definition 4.2 . we have $\phi_{i t}\left(D^{\#} f^{s}\left(\alpha_{j}\right)\right)=\angle\left(D f^{s} E_{i}, D f^{s} E_{t}\right)$, which does not depend on the choice of $j$. Thus

$$
\begin{aligned}
\lim _{n \rightarrow+\infty} & \frac{1}{p_{n}} \sum_{s=0}^{p_{n}-1} \angle\left(D f^{s} E_{i}\left(z_{n}\right), D f^{s} E_{t}\left(z_{n}\right)\right) \\
= & \lim _{n \rightarrow+\infty} \frac{1}{p_{n}} \sum_{s=0}^{p_{n}-1} \phi_{i t}\left(D^{\#} f^{s} \alpha_{z_{n}}\right) \\
= & \sum_{j=1}^{2^{d}} a_{j}\left(\lim _{n \rightarrow+\infty} \frac{1}{n} \sum_{s=0}^{n-1} \angle\left(D f^{s} E_{i}, D f^{s} E_{t}\right)\right) \\
= & \lim _{n \rightarrow+\infty} \frac{1}{n} \sum_{s=0}^{n-1} \angle\left(D f^{s} E_{i}, D f^{s} E_{t}\right) \\
= & m \angle_{\tilde{\omega}}\left(E_{i}, E_{t}\right) .
\end{aligned}
$$

So the mean angles between the Oseledec invariant bundles $E_{i}\left(z_{n}\right)$ and $E_{t}\left(z_{n}\right)$ converge to the mean angle between the Oseledec invariant bundles $E_{i}$ and $E_{t}$. We have proved Theorem $1.12(1)$.

Proof of Theorem 1.12(2). We recall the measures $\omega_{n}$ and $\tilde{\omega}$ in (a)(b) and $\mu_{n}$ and $\mu$ in the proof of (1) and their relations:

$$
\omega_{n} \rightarrow \tilde{\omega}, \quad \mu_{n} \rightarrow \mu, \quad \pi_{*} \mu_{n}=\omega_{n}, \quad \pi_{*} \mu=\tilde{\omega} .
$$

Since the function $\tau: \mathcal{U}_{d}^{\#} \rightarrow \mathbb{R}$ is continuous by Proposition 2.6 $\int \tau d \mu_{n} \rightarrow \int \tau d \mu$. Since all the ergodic measures decomposed from $\mu$ have the same type, we have that

$$
\int \tau d \mu=\int \tau d \tilde{\omega}
$$

Note that $\omega_{n}$ is an ergodic measure with simple spectrum. This implies that

$$
\int \tau d \mu_{n}=\int \tau d \omega_{n}
$$

as shown in the explanation below (1.2). Hence we have that $\int \tau d \omega_{n} \rightarrow \int \tau d \tilde{\omega}$ or $\tilde{\tau}\left(\omega_{n}\right) \rightarrow \tilde{\tau}(\tilde{\omega})$. We thus obtain $(2)$.

Proof of Theorem [1.12(3). Take $k$ large, so that

$$
\tilde{\omega}\left(\Lambda_{k}(\tilde{\omega})\right)>1-\varepsilon,
$$

where $\Lambda_{k}(\tilde{\omega})$ denotes the $k$-th Pesin block associated with $\tilde{\omega}$. Since the splitting

$$
x \rightarrow E_{1}(x) \oplus \cdots \oplus E_{d}(x)
$$


depends continuously on $x \in \Lambda_{k}(\tilde{\omega})$, we can choose a uniform constant $\eta>0$ satisfying

$$
\frac{\eta<\min _{i \neq j}}{x \in \Lambda_{k}(\tilde{\omega})}\left\{\frac{1}{10} \angle\left(E_{i}(x), E_{j}(x)\right), \varepsilon\right\} .
$$

For each $x \in \Lambda_{k}(\tilde{\omega}) \cap \operatorname{Supp}(\tilde{\omega})$, we take and fix $\alpha_{0}(x)=\left(u_{1}, \ldots, u_{d}\right) \in$ $Q_{\mu}\left(\mathcal{U}_{d}^{\#}(x), D^{\#} f\right) \cap \operatorname{Supp}(\mu)$, where $\mu$ is the measure taken in the proof of $(1)$. Denote by $U\left(\alpha_{0}(x), \eta\right)$ the $\eta$-neighborhood of $\alpha_{0}(x)$ under the metric on the Grassman bundle. Then $\mu\left(U\left(\alpha_{0}(x), \eta\right)\right)>0$. Recalling that $\mu_{n} \rightarrow \mu$, we have

$$
\liminf _{n \rightarrow+\infty} \mu_{n}\left(U\left(\alpha_{0}(x), \eta\right)\right) \geq \mu\left(U\left(\alpha_{0}(x), \eta\right)\right)>0 .
$$

Therefore, we can take an integer $N(x)=N\left(\alpha_{0}(x)\right)>0$ such that

$$
\mu_{n}\left(U\left(\alpha_{0}(x), \eta\right)\right)>0, \quad \forall n \geq N(x) .
$$

This implies the existence of a frame $\beta_{n}(x)$ in $Q_{\mu_{n}}\left(\mathcal{U}_{d}^{\#}, D^{\#} f\right) \cap \operatorname{supp}\left(\mu_{n}\right) \cap$ $U\left(\alpha_{0}(x), \eta\right)$ for each $n \geq N(x)$. Observing that $\mu_{n}$ covers the atomic measure $\omega_{n}$, we can deduce that $\beta_{n}(x)$ must be a frame based on a periodic point on $\operatorname{Orb}\left(z_{n}\right)$, where $z_{n}$ is the periodic point chosen in (a)(b). Since $\omega_{n}$ is a hyperbolic measure with simple spectrum by Theorem 1.9 , we have by the claim before Definition 4.2 that each vector in $\beta_{n}(x)$ must be in a subbundle of the Oseledec splitting of $\omega_{n}$ and any two vectors lie in different subbundles. Thus the Oseledec splitting of $\tilde{\omega}$ at $x$ is $\eta$ closed by the Oseledec splitting of $\omega_{n}$ at a point $z=z(x, n)$ on $\operatorname{Orb}\left(z_{n}\right), n \geq N(x)$. Note that the number $N(x)$ may vary with $x$; we need to find a number $\tilde{N}$, independent of the choice of $x \in \Lambda_{k}(\tilde{\omega})$, such that $\mu_{\tilde{N}}$ meets $(3)$. This can be done by the compactness of $\Lambda_{k}(\tilde{\omega})$ and continuity of the Oseledec splitting on it.

Hence we complete the proof of Theorem 1.12 .

Proof of Theorem 1.13. (a) is from Katok's closing lemma [4]. (b) is from Theorem 1.9. (c) is from Theorem 1.8. Combining (a)-(c) with Theorem 1.12, one can deduce (d)-(f) immediately.

\section{ACKNOWLEDGEMENT}

The authors thank the referee for his/her suggestions for improving the manuscript.

\section{REFERENCES}

[1] J. Bochi, Genericity of zero Lyapunov exponents, Erg. Th. Dyn. Sys. 22 (2002), 1667-1696. MR.1944399 (2003m:37035)

[2] X. Dai, Liao style numbers of differential systems, Communication Cont. Math., Vol. 6, (2004), 279-299. MR2057843 (2005c:37026)

[3] M. Hirayama, Periodic probability measures are dense in the set of invariant measures, Dis. Cont. Dyn. Sys. 9 (2003), 1185-1192. MR1974422(2004a:37032)

[4] A. Katok, Liapunov exponents, entropy and periodic orbits for diffeomorphisms, Pub. Math. IHES 51 (1980), 137-173. MR573822 (81i:28022)

[5] A. Katok, L. Mendoza, Dynamical systems with non-uniformly hyperbolic behavior, Supplement to the book: A. Katok, B. Hasselblatt, Introduction to the modern theory of dynamical systems, Cambridge Univ. Press, USA (1995). MR1326374 (96c:58055) 
[6] S. T. Liao, Certain ergodic theorems for differential systems on a compact differentiable manifold, Acta Sci. Natur. Univ. Pekin. 9 (1963), 241-265, 309-327 (in Chinese). English translation appears as Chapter 1 in Liao's book "Qualitative Theory on Differentiable Dynamical Systems," Science Press, Beijing, New York (1996). MR2225398 (2007e:37014)

[7] R. Mañé, Ergodic theory and differentiable dynamics, Springer-Verlag (1987). MR889254 (88c:58040)

[8] B. B. Nemytskii, B. B. Stepanov, Qualitative theory of differential equations, Princeton University Press (1960). MR0121520 (22:12258)

[9] V. I. Oseledec, Multiplicative ergodic theorem, Liapunov characteristic numbers for dynamical systems, Trans. Moscow Math. Soc. 19 (1968), 197-221; translated from Russian. MR0240280 (39:1629)

[10] J. Palis, W. de Melo, Geometric theory of dynamical systems, an introduction, SpringerVerlag (1982). MR669541 (84a:58004)

[11] Y. Pesin, Lyapunov characteristic exponents and ergodic properties of smooth dynamical systems with an invariant measure, Sov. Math. Dok. 17 (1976), 196-199.

[12] Y. Pesin, Families of invariant manifolds corresponding to nonzero Liapunov exponents, Izvestija 10 (1976), 1261-1305.

[13] M. Pollicott, Lectures on ergodic theory and Pesin theory on compact manifolds, Cambridge Univ. Press (1993). MR1215938 (94k:58080)

[14] K. Sigmund, On dynamical systems with the specification property, Trans. Amer. Math. Soc. 190 (1974), 285-299. MR0352411(50:4898)

[15] W. Sun, Characteristic spectrum for differential systems, Jour. Diff. Equa. 147 (1998), 184194. MR 1632673 (2000e:37016)

[16] W. Sun, Characteristic spectra for parallelotope cocycles, Dynamical Systems, World Scientific, Singapore (1999), 256-265.

[17] W. Sun, E. Vargas, Entropy and ergodic probability for differentiable dynamical systems and their bundle extensions, Topology and its Application 154 (2007), 683-697. MR2280913

[18] Z. Wang, W. Sun, Lyapunov exponents of hyperbolic measures and hyperbolic periodic orbits, preprint (2005).

[19] Z. Zhou, Weakly almost periodic points and measure center, Science in China A (1992), 572-581. MR1223083 (94f:58082)

[20] Z. Zhou, Measure center and minimal abstracting center, Bull. Science in China 37 (1992), 2115-2118.

Lmam, School of Mathematical Sciences, Peking University, Beijing 100871, PeoPle's Republic of China - and - Applied Mathematical Department, The Central University of Finance and Economics, Beijing 100081, People's Republic of China

E-mail address: Chaol@math.pku.edu.cn

E-mail address: Chaol@cufe.edu.cn

Lmam, School of Mathematical Sciences, Peking University, Beijing 100871, PeoPle's Republic of China - and - China Institute of Advanced Study, The Central University of Finance and Economics, Beijing 100081, People's Republic of China

E-mail address: yaseplay@gmail.com

Lmam, School of Mathematical Sciences, Peking University, Beijing 100871, PeoPLE'S Republic of ChinA

E-mail address: sunwx@math.pku.edu.cn 